TRANSACTIONS OF THE

AMERICAN MATHEMATICAL SOCIETY

Volume 365, Number 7, July 2013, Pages 3437-3476

S 0002-9947(2013)05629-2

Article electronically published on February 7, 2013

\title{
LARGE DEVIATION ESTIMATES FOR SOME NONLOCAL EQUATIONS. GENERAL BOUNDS AND APPLICATIONS
}

\author{
CRISTINA BRÄNDLE AND EMMANUEL CHASSEIGNE
}

ABSTRACT. Large deviation estimates for the following linear parabolic equation are studied:

$$
\frac{\partial u}{\partial t}=\operatorname{Tr}\left(a(x) D^{2} u\right)+b(x) \cdot D u+\mathcal{L}[u](x),
$$

where $\mathcal{L}[u]$ is a nonlocal Lévy-type term associated to a Lévy measure $\mu$ (which may be singular at the origin):

$$
\mathcal{L}[u](x)=\int_{\mathbb{R}^{N}}\left\{\left(u(x+y)-u(x)-(D u(x) \cdot y) \mathbf{I}_{\{|y|<1\}}(y)\right\} \mathrm{d} \mu(y) .\right.
$$

Assuming only that some negative exponential integrates with respect to the tail of $\mu$, it is shown that given an initial data, solutions defined in a bounded domain converge exponentially fast to the solution of the problem defined in the whole space. The exact rate, which depends strongly on the decay of $\mu$ at infinity, is also estimated.

\section{INTRODUCTION}

The problem we consider in this paper is related to the following parabolic nonlocal equation:

$(1.1)$

$\frac{\partial u}{\partial t}=\operatorname{Tr}\left(a(x) D^{2} u\right)+b(x) \cdot D u+\int_{\mathbb{R}^{N}}\left\{\left(u(x+y)-u(x)-(D u(x) \cdot y) \mathbb{I}_{\{|y|<1\}}(y)\right\} \mathrm{d} \mu(y)\right.$,

associated to an initial data $u(x, 0)=u_{0}$ in $\mathbb{R}^{N}$. Here, the functions $u_{0}: \mathbb{R}^{N} \rightarrow \mathbb{R}$, $a: \mathbb{R}^{N} \rightarrow \mathbb{R}$ and $b: \mathbb{R}^{N} \rightarrow \mathbb{R}^{N}$ are continuous and bounded, $a \geqslant 0$ and $\mu$ is a Lévy measure, that is, it is a Borel measure such that $\int \inf \left(1,|z|^{2}\right) \mathrm{d} \mu(z)<\infty$. For simplicity we shall also assume that the measure $\mu$ has a density, noted by $J$, with respect to the Lebesgue measure in $\mathbb{R}^{N}$.

Recently, equations such as (1.1) involving a Lévy-type nonlocal term

$$
\mathcal{L}[u](x):=\int_{\mathbb{R}^{N}}\left\{\left(u(x+y)-u(x)-(D u(x) \cdot y) \mathbb{I}_{\{|y|<1\}}(y)\right\} \mathrm{d} \mu(y)\right.
$$

have been under thorough investigation by many authors and in many directions. Indeed, they present challenging problems which are not covered by the existing "local" vision of pde's. References are too many to cite; those which are more closely related to our concern are given below.

Received by the editors April 14, 2010 and, in revised form, May 12, 2011.

2010 Mathematics Subject Classification. Primary 47G20, 60F10; Secondary 35A35, 49L25.

Key words and phrases. Nonlocal diffusion, large deviation, Hamilton-Jacobi equation, Lévy operators.

(C)2013 American Mathematical Society Reverts to public domain 28 years from publication 
Our main goal here is the following: we want to obtain some estimates on how the solutions $u_{R}$ that are defined in the ball $B_{R}=\{|x|<R\}$ approach the solution $u$ in all $\mathbb{R}^{N}$ as $R \rightarrow \infty$, in the spirit of [6]. To be more precise, let $u_{R}$ be the solution of the following Cauchy-Dirichlet problem:

$$
\begin{cases}\frac{\partial u}{\partial t}=\operatorname{Tr}\left(a(x) D^{2} u\right)+b(x) \cdot D u+\mathcal{L}[u](x) & \text { in } B_{R} \times(0, \infty), \\ u(x, t)=0 & \text { in }\left(\mathbb{R}^{N} \backslash B_{R}\right) \times(0, \infty), \\ u(x, 0)=u_{0}(x) & \text { in } B_{R} .\end{cases}
$$

Then, to briefly sum up the main results of the paper, we show that provided the measure $\mu$ has at most an exponential tail, a bound of the following kind (see more precise formulations below) always holds as $R \rightarrow \infty$ :

$$
\left|u-u_{R}\right|(x, t) \leqslant \mathrm{e}^{-R f(\ln R)(1+o(1))},
$$

where $o(1)$ is a function which goes to zero as $R \rightarrow 0$, independently of $u$. The behaviour of $f: \mathbb{R}_{+} \rightarrow \mathbb{R}_{+}$depends on the tail of $\mu$, but in any case $f$ cannot grow faster than linearly and is at least bounded below by some positive constant near infinity under our assumptions.

This has to be compared to the classical $\exp \left(-R^{2}\right)$-type estimate associated to the heat equation [6]: here, the presence of nonlocal terms implies an order $\exp (-R \ln R)$ at best, as we shall see.

Such a result is obviously related to a Large Deviation Principle (LDP for short) for the associated Lévy process; see below for more detail.

Typical examples. As for (1.1), a first example we have in mind is the convolution equation,

$$
\frac{\partial u}{\partial t}=J * u-u
$$

Here $a, b=0$ and $\mu$ is a Lévy measure with probability density $J$, which is symmetric and integrable; see [18, 19. In [12, the authors gave partial answers for this equation when $J$ decays strictly faster than an exponential at infinity. Our results here include and generalize those given in 12. Be aware that writing the equation under the form (1.5) requires $J$ to be of unit mass, and moreover that in this case $\mathrm{d} \mu(z)=J(-z) \mathrm{d} z$, a detail which has to be taken into account when dealing with nonsymmetric kernels.

A more sophisticated example is the following:

$$
\frac{\partial u}{\partial t}=\Delta u+b \cdot D u+\text { P.V. } \int_{\mathbb{R}^{N}}\{u(x+y, t)-u(x, t)\} \frac{\mathrm{e}^{-|y|}}{|y|^{N+\alpha}} \mathrm{d} y,
$$

where P.V. stands for principal value. This is the same as putting the compensator term $D u(x) \cdot y$ since the measure density $J(y)=\mathrm{e}^{-|y|} /|y|^{N+\alpha}$ is symmetric. This kind of measure, called tempered $\alpha$-stable law, appears for instance in finance [34.

It is important to notice that our framework (which will be fully established in Section 2) does not allow us to treat fractional Laplace-type nonlocal terms for which $J(y)=1 /|y|^{N+\alpha}, \alpha \in(0,2)$, since we require the tail of $J$ to go to zero at least exponentially fast.

Existence and uniqueness of solutions. Concerning the existence and uniqueness of solutions of (1.1), first notice that in the case of constant coefficients $a$ and $b$, this can be derived from a Fourier analysis of the equation. In the absence of differential terms, i.e., $a=b=0$ and if $\mu$ is a finite measure, various 
results about existence and uniqueness of solutions in $\mathbb{R}^{N}$ or in bounded domains can be found in $[12,13,18,19]$.

In the case when $a(x)=\sigma \sigma^{t}$ with $\sigma$ and $b$ Lipschitz, existence and uniqueness of bounded solutions are proven in 8 for the elliptic (stationary) version of the equation under some assumptions on $\mu$. With little modifications (which essentially amount to changing the $(-\gamma u)$-term in the equation for a $u_{t}$-term), the same result holds true for the parabolic version, and gives existence and uniqueness of solutions in $\mathbb{R}^{N}$ - (1.1), or in a bounded domain - (1.3). See also [1, 15, 16] for some other results.

Here we shall not derive a full theory of existence, uniqueness and comparison for (1.1) or (1.3) which is not the central point of this paper. Rather, we will simply assume that given an initial data $u_{0}$ continuous, positive and bounded, there exists a unique viscosity solution $u(x, t)$ of (1.1) with initial data $u_{0}$. We also consider for any $R>0$ problem (1.3) and will assume that there exists a unique solution $u_{R}$ to this problem. Let us mention that the possible incompatibility of the initial and boundary data at $|x|=R, t=0$ can be overcome by using an approximation with a sequence $u_{0} \chi_{n}$, where $\chi_{n}$ is smooth and converges to the indicator function of the ball $B_{R}$. Starting from this, the paper is devoted to estimating the difference $\left(u-u_{R}\right)$.

Lévy processes and the Large Deviation Principle. Concerning Lévy processes, we shall not enter into details here since it is not the aim of the paper and refer for instance to 2, 11, 20, for a probability-oriented approach, but let us just mention a few facts.

A Lévy process is a stochastic cadlag process $\left(X_{t}\right)_{t \geqslant 0}$ which has stationary and independent increments: for any $0<s<t,\left(X_{t}-X_{s}\right)$ only depends on $(t-s)$, and the increments $\left(X_{t_{i+1}}-X_{t_{i}}\right)$ are independent for nonoverlapping time intervals. An important fact is that $\left(X_{t}\right)$ is not required to be continuous (in time), so that this type of process can model either continuous diffusions or jump diffusions, and a mix of both. Thus, typical Lévy processes are the "usual" Brownian motion, the compound Poisson process and the $\alpha$-stable jump process.

Actually, the Lévy-Khintchine formula shows that a Lévy process (actually, any infinitely divisible law) can be decomposed as the sum of a Brownian motion, a drift and a pure jump process. The continuous (Brownian) part is associated to the Laplacian-type diffusion term $\operatorname{Tr}\left(a(x) D^{2} u\right)$, while a drift corresponds to the $b(x) \cdot D u$-term. In the case of the compound Poisson process, the nonlocal term can be rewritten as $J * u-u$, leading to (1.5). Finally, in the case of the $\alpha$-stable law, $J(z)=|z|^{-N-\alpha}$ and we recover the well-known fractional Laplace operator. A complementary approach, using the Feller semi-group associated to a Lévy process $\left(X_{t}\right)$, implies that the infinitesimal generator of the semi-group has exactly the form of the right-hand side of (1.1).

Estimate (1.4) may be considered in the framework of large deviation principles. These kinds of results go back to Cramér in 1938, see [22, but the large deviations theory has been developed a lot since then; see for instance the works of WentzellFreidlin [38, and Donsker-Varadhan [24. For more recent references, we refer to the monographs [28, 31] and [37. For a more pde-oriented approach, which is the one we use here, Fleming [27] was probably the first to use the log transform. The viscosity solutions' framework was first employed by Evans and Ishii in 1985; see [25]. 
A sequence of probability measures $\left(\mathbb{P}_{n}\right)$ on a polish space $X$ is said to satisfy an LDP with speed $\left(a_{n}\right), a_{n} \rightarrow \infty$, and l.s.c. rate function $I: X \rightarrow \mathbb{R}_{+}$if for any Borelian set $E \subset X$, we have

$$
-\inf _{x \in \operatorname{int}(E)} I(x) \leqslant \liminf _{n \rightarrow \infty} \frac{1}{a_{n}} \ln \mathbb{P}_{n}(E) \leqslant \limsup _{n \rightarrow \infty} \frac{1}{a_{n}} \ln \mathbb{P}_{n}(E) \leqslant-\inf _{x \in \operatorname{clo}(E)} I(x),
$$

where $\operatorname{int}(E), \operatorname{clo}(E)$ stand for the interior and the closure of $E$, respectively.

Here we prove that

$$
\limsup _{R \rightarrow \infty} \frac{1}{R} \ln \left[\left(u-u_{R}\right)(R x, R t)\right]=-I_{\infty}(x, t)
$$

in $B_{1} \times(0, \infty)$, where the rate function $I_{\infty}$ is identified as the unique viscosity solution of a Hamilton-Jacobi equation (1.6). So, it is indeed an LDP-type estimate where $R \rightarrow \infty$ plays the role of the speed $\left(a_{n}\right)$.

Of course this is only one of the estimates, so we do not pretend that we have an LDP stricto sensu. To do so we would need to consider a rescaled process in $B_{1}$ associated to $\left(u-u_{R}\right)(R x, R t)$ and make a precise analysis from the probabilistic viewpoint, which we do not pretend to do here. Nevertheless, let us notice that $\left(u-u_{R}\right)(x, T)$ measures the probability of escaping the ball $B_{R}$ between time $t=0$ and $t=T$ when starting from $x$. This assertion is made precise by using a Feynman-Kac formula for $u$ and $u_{R}$; see [2, Chapter 6]. More precisely, let us consider the following stochastic differential equation associated to the jump diffusion $\left(X_{t}\right)$, where $B_{t}$ is a Brownian motion:

$$
\mathrm{d} X_{t}=b\left(X_{t^{-}}\right) \mathrm{d} t+\sigma\left(X_{t^{-}}\right) \mathrm{d} B_{t}+\int_{|x|<1} x \tilde{N}(\mathrm{~d} x, \mathrm{~d} t)+\int_{|x| \geqslant 1} x N(\mathrm{~d} x, \mathrm{~d} t) .
$$

Here, $N$ is a Poisson random measure independent of $B$ and $\tilde{N}$ is the associated compensated Poisson random measure. Let us denote by $\left(X_{t}^{x}\right)$ the solution associated to the initial condition $X_{0}^{x}=x$ almost everywhere in $\mathbb{R}^{N}$. Then the solution of (1.1) can be calculated as follows: $u(x, t)=\mathbb{E}^{x}\left(u_{0}\left(X_{t}^{x}\right)\right)$, where $\mathbb{E}^{x}$ denotes the conditional expectation with respect to the event $\left\{X_{0}^{x}=x\right\}$ and $a=\sigma \sigma^{t}$. In the same way, the solution of (1.3) can be expressed thanks to the first exit time from the ball $B_{R}, \tau_{R}(x):=\inf \left\{t \geqslant 0: X_{t}^{x} \notin B_{R}\right\}$ as $u_{R}(x, t)=\mathbb{E}^{x}\left(u_{0}\left(X_{t}^{x}\right) \mathbb{I}_{\left\{\tau_{R} \geqslant t\right\}}\right)$. Then, it follows that

$$
\left(u-u_{R}\right)(x, T)=\mathbb{E}^{x}\left(u_{0}\left(X_{T}^{x}\right) \mathbf{I}_{\left\{\tau_{R}<T\right\}}\right)
$$

and taking for instance $u_{0}=1$, we obtain that $\left(u-u_{R}\right)(x, T)=\mathbb{E}^{x}\left(\mathbb{I}_{\left\{\tau_{R}<T\right\}}\right)$, which is exactly the probability of escaping the ball $B_{R}$ before time $T>0$.

Hence, our estimates prove that as $R \rightarrow \infty$, we have an exponentially small probability to escape $B_{R}$ in a finite time interval.

The general strategy. Let us sketch the strategy adapted from [6] which is used to get the exponential estimates (1.4): it consists in first scaling the equation in order to obtain a problem in a fixed domain, then rewriting the equation for the $\log$ transform. Namely here, we consider the solution $v_{R}$ of (1.3) in $B_{R} \times(0, \infty)$ with initial value $v_{R}(x, 0)=0$ for $x \in B_{R}$ and "boundary data" $v_{R}=\left\|u_{0}\right\|_{\infty}$ for $|x| \geqslant R$. By comparison, $0 \leqslant u-u_{R} \leqslant v_{R}$ so that we need only to estimate $v_{R}$. 
Then we consider

$$
I_{R}(x, t):=-\frac{1}{R} \ln v_{R}(R x, R t) \quad \text { in } \quad B_{1} \times[0, \infty)
$$

and we prove that $I_{R}$ converges to the unique viscosity solution of the following Hamilton-Jacobi equation:

$$
\begin{cases}\partial_{t} I_{\infty}+H\left(D I_{\infty}\right)=0 & \text { in } B_{1} \times(0, \infty), \\ I_{\infty}=0 & \text { on } \partial B_{1} \times(0, \infty), \\ I_{\infty}(x, 0)=+\infty & \text { in } B_{1} .\end{cases}
$$

The Hamiltonian associated to (1.1) is given by:

$$
H(p):=\operatorname{Tr}\left(a_{\infty} p \otimes p\right)+b_{\infty} \cdot p+\int_{\mathbb{R}^{N}}\left(\mathrm{e}^{p \cdot y}-1-(p \cdot y) \mathbb{I}_{\{|y|<1\}}(y)\right) \mathrm{d} \mu(y),
$$

where $a_{\infty}$ and $b_{\infty}$ are defined as the limits as $|x| \rightarrow \infty$ of $a$ and $b$, respectively. The Lagrangian $L$ associated to $H$ is defined as usual as

$$
L(q):=\sup _{p \in \mathbb{R}^{N}}\{p \cdot q-H(p)\},
$$

and using a Lax-Oleinik formula, see 33, we obtain a semi-explicit expression for $I_{\infty}$, see (3.2), in terms of $L$ and depending on the tail of $J$. This in turn gives us the exponential estimates for $\left(u-u_{R}\right)$ after coming back to the original variables.

Surprisingly, the scaling which has to be used here is of hyperbolic type: $(R x, R t)$, contrary to what happens for the heat equation where the parabolic scaling $(R x$, $R^{2} t$ ) is performed (see [6]). This deserves some discussion: more general scalings of the form $\left(R x, R^{\sigma} t\right)$ can be considered, but if we want to track the large deviation principle and use a $\log$ transform such as $s \mapsto(1 / R)^{\gamma} \ln (s)$, it is easily seen that in order to get a nontrivial Hamilton-Jacobi equation in the limit, the choice $\sigma=\gamma=1$ is required. However, we refer to [28, Chapter 10] where a different time scale is also considered which kills the nonlocal term and tracks the central limit theorem instead of the Large Deviation Principle. See Remark 3.2 for more details.

In the same spirit, it would be interesting to have an estimate of $\left(u-u_{R}\right)$ in the case of the fractional Laplacian, $J(y)=|y|^{-(N+\alpha)}$ where $\alpha \in(0,2)$. However, since the tail of such a $J$ does not decay exponentially fast, it is mostly improbable that in such a case the Large Deviation Principle holds. By this we mean that the rate of convergence of $u_{R}$ to $u$ should not be exponentially small because there are "many" long jumps that allow the process to escape $B_{R}$. On the contrary, there are many hints that indicate a power-like estimate should hold. Thus, in this case it seems natural to try a scaling of the form $\left(R^{\sigma} x, R t\right)$, in conjunction with the transform $s \mapsto(1 / R)^{\gamma} s^{\beta}$. However, it is still unclear to us how to obtain a proof and identify the associated rate function. We hope to answer this interesting question in a future work.

In our present framework, obtaining (1.6) is more involved than in [12], since we have to deal with the differential terms and the singular part in $H$. Hence, we face several difficulties that come from the generalization of the equation, which imply nontrivial adaptations and new developments of some aspects of the theory. Let us briefly explain the main points we have to deal with:

(i) Singular measures at the origin: using kernels with singularities requires first a suitable concept of a solution; here we use the notion of viscosity solutions derived in [7] for Lévy-type operators, which allows us to handle 
this situation. Then we introduce what we call the essential Hamiltonian, $H^{\text {ess }}$, wiping out the singularity at the origin, see Definition 2.4 and prove that asymptotically $H$ and $H^{\text {ess }}$ are equivalent (and the same holds for the associated Lagrangians).

(ii) Nonsymmetric kernels: we want to estimate $L$ in order to obtain an expression for $I_{\infty}$. This needs an extra effort in this case: since we do not have symmetry, the vectors $p$ and $D H(p)$ do not necessarily point in the same direction. Hence dot products cannot be simplified and we have to carefully analyze the angle between vectors. We prove some results valid for nonsymmetric kernels $J$ at the logarithm level, and others using the smallest symmetric kernel above $J$.

(iii) Differential terms: the first two terms in equation (1.1) do not introduce much difficulty with respect to [12, but we have to deal with them with the notion of a viscosity solution. To be able to pass to the limit, we will assume that $a$ and $b$ have limits at infinity.

(iv) Possibly infinite Hamiltonians: in the case when $J(y) \sim \mathrm{e}^{-\alpha|y|}$ as $|y| \rightarrow$ $\infty, H$ is infinite outside $B_{\alpha}$, which makes the analysis of (1.6) much more delicate, since an initial layer appears. Thus, one of the major contributions of this paper is to provide existence, uniqueness and comparison results for the Hamilton-Jacobi equation $u_{t}+H(D u)=0$ when $H$ is infinite outside the ball.

Main results. First we have a general result, Theorem 3.1, which gives a theoretical bound in terms of the rate function $I_{\infty}$, typical of large deviation estimates: as $R \rightarrow \infty$,

$$
\left|u-u_{R}\right|(x, t) \leqslant \mathrm{e}^{-R I_{\infty}(x / R, t / R)+o(1) R} .
$$

Then we concentrate our efforts in finding a behaviour for $L$, which allows us to give more explicit convergence rates for $u-u_{R}$, depending on the tail of $J$. We distinguish three cases, namely compactly supported kernels, and what we call intermediate and critical kernels.

- We first deal with compactly supported measures in Section 4. As was shown in 12 for symmetric, compactly supported measures, the rate of convergence is of type $R \ln R$. As we will see, cf. Theorem 4.3, the same holds for general nonsymmetric and singular kernels: for any $\theta \in(0,1)$ and $T>0$, as $R \rightarrow \infty$,

$$
\sup _{\substack{|x| \leqslant \theta R \\ 0 \leqslant t \leqslant T R}}\left|u-u_{R}\right|(x, t) \leqslant \mathrm{e}^{-(1-\theta) R \ln R+o(R \ln R)} .
$$

- We also consider what we call critical kernels, for which $H$ is not finite everywhere. This is done in Section [6] and we devote a large part of this section to treating the Hamilton-Jacobi equation with such an $H$. The main theorem in this section states that in this case the rate of convergence is linear (see Theorem 6.9): for any $\theta \in(0,1)$ and $T>0$, as $R \rightarrow \infty$,

$$
\sup _{\substack{|x| \leqslant \theta R \\ 0 \leqslant t \leqslant T R}}\left|u-u_{R}\right|(x, t) \leqslant \mathrm{e}^{-(1-\theta) R+o(R)}
$$

The proof is essentially the same as the one for compactly supported kernels; however the analysis of the Hamilton-Jacobi equation is much more involved since, as 
was said, infinite values of the Hamiltonian imply an initial layer that has to be carefully analyzed.

- In between compactly supported and critical measures we consider, see Section 5 the fast decaying (or intermediate) measures. For these kernels the convergence rate is not explicit in general, save for some special cases (see Section 7) but it can be expressed through the Legendre transform, $\mathcal{K}$, of $\ln (1 / J)$. In a simplified way, assuming that $J$ is symmetric (see Theorem 5.9 for more details and the general version) the convergence rate reads: for any $\theta \in(0,1)$ and $T>0$, as $R \rightarrow \infty$,

$$
\sup _{\substack{|x| \leqslant \theta R \\ 0 \leqslant t \leqslant T R}}\left|u-u_{R}\right|(x, t) \leqslant \mathrm{e}^{-(1-\theta) R \mathcal{K}^{-1}(\ln ((1-\theta) R / t))(1+o(1))} .
$$

See Section 7 for some explicit examples of rates.

An estimate for a nonlocal KPP problem. As an interesting by-product, the techniques we develop here can be applied to also obtain estimates for the following nonlocal version of the Kolmogorov-Petrovskii-Piskounov equation:

$$
\partial_{t} u-\operatorname{Tr}\left(a(x) D^{2} u\right)-b(x) \cdot D u-\mathcal{L}[u](x)=u(1-u) \quad \text { in } \quad \mathbb{R}^{N} \times[0, T]
$$

with a continuous initial data $u_{0}(x)=g(x), 0 \leqslant g \leqslant 1$. The original equation corresponds to the case $a=1, b=0$ and especially does not contain the nonlocal term $\mathcal{L}$; see 32 . It is well known that the solution $u$ converges to the equilibrium states 0 and 1 as $x$ and $t$ go to infinity. M. Freidlin studied this phenomenon from the probability and large deviations point of view [29, 30 and a pde approach was developed in [26]. Following the ideas in [5], we are also able to adapt our results to derive exponential rates of convergence towards the equilibrium states; see Section 7.3 for more on this subject. Here also, the interesting case of the fractional Laplacian unfortunately needs to be left out, since our results require the tail of $\mu$ to be at most exponential. But again, it is not clear at all that, in such a case, the convergence should be exponentially fast. The interested reader will find further references about this equation and traveling waves in the works [21] and [14.

To end this introduction, we mention that further comments and results are also to be found in Section 7? we give some explicit calculations of rates and consider totally asymmetric measures in 1-D. Such asymmetric measures are also used in finance; see for instance the CGMY model [17.

Notation. Throughout the paper we will denote by $\left(e_{i}\right)$ the canonical basis of $\mathbb{R}^{N}$, while $\nu$ will refer to any unit vector. The Euclidean scalar product is denoted as $a \cdot b$ and we use $|\cdot|$ for the associated norm in $\mathbb{R}^{N}$. We will use ang $(a, b)$ to denote the angle between the vectors $a$ and $b$ (see Definition 5.4) and $a \rightarrow a^{T}$ is the transposition. The notation $B(x, r)$ stands, as is usual, for the ball of radius $r>0$, centered at $x \in \mathbb{R}^{N}$ and we also use the simpler notation $B_{r}=B(0, r)$. We also denote by $D u \in \mathbb{R}^{N}$ the gradient of $u$ and by $D^{2} u \in \mathcal{M}_{N}(\mathbb{R})$ the Hessian matrix of $u$. In all of the paper, $c(J)$ denotes any constant which only depends on $J$, possibly varying from one line to another. The notation $J_{1} \prec J_{2}$ essentially means that $J_{1} \leqslant J_{2}$ in $\mathbb{R}^{N}$, but we add some strict separation inside a small region (see Definition 2.9). If $a, b \in \mathbb{R}$, we denote by $a \wedge b$ the infimum of $a$ and $b$. 


\section{Preliminaries}

Now let us focus on the concrete hypotheses that concern the equations we have in mind. For $\mu, a, b, a_{\infty}, b_{\infty}$ in (1.1) and (1.7) there are essentially three assumptions we shall make throughout the paper:

Hypothesis 1. The measure $\mu$ is a Lévy measure with density $J(\cdot)$ satisfying

$$
\left\{\begin{array}{l}
J: \mathbb{R}^{N} \backslash\{0\} \rightarrow \mathbb{R}_{+} \text {is } C^{1} \text {-smooth, } \\
\exists \rho_{0}>0, \operatorname{supp}(J) \supset B_{\rho_{0}}, \\
\int_{\mathbb{R}^{N}}\left(1 \wedge|y|^{2}\right) J(y) \mathrm{d} y<\infty .
\end{array}\right.
$$

Hence in the sequel we rewrite (1.7) as

$$
H(p):=\operatorname{Tr}\left(a_{\infty} p \otimes p\right)+b_{\infty} \cdot p+\int_{\mathbb{R}^{N}}\left(\mathrm{e}^{p \cdot y}-1-(p \cdot y) \mathbf{I}_{\{|y|<1\}}(y)\right) J(y) \mathrm{d} y .
$$

The last assumption in (2.1) implies in particular that the integral in (2.2) over $\{|y|<1\}$ converges.

Hypothesis 2. The $N \times N$-matrix $a(\cdot)$ is continuous and nonnegative, $b(\cdot)$ is a continuous vector field in $\mathbb{R}^{N}$ and we also assume that the following limits are well-defined:

$$
a_{\infty}=\lim _{|x| \rightarrow \infty} a(x) \in \mathcal{M}_{n}(\mathbb{R}), \quad b_{\infty}=\lim _{|x| \rightarrow \infty} b(x) \in \mathbb{R}^{N}
$$

(notice that necessarily $a_{\infty}$ is nonnegative).

Except in Section [6 we also make the following assumption on $J$ :

Hypothesis 3.

$$
\forall \beta>0, \quad \int_{\left\{|y|>\rho_{0} / 2\right\}} \mathrm{e}^{\beta|y|} J(y) \mathrm{d} y<\infty .
$$

This ensures that the exponential part of the Hamiltonian in (1.7) (or (2.2) ) converges for any $p \in \mathbb{R}^{N}$. In Section 6 , we shall only assume that

$$
\sup \left\{\beta>0: \int_{\left\{|y|>\rho_{0} / 2\right\}} \mathrm{e}^{\beta|y|} J(y) \mathrm{d} y<\infty\right\}=\beta_{0}>0,
$$

which is the case for exponential-type tails, and implies that the associated Hamiltonian is not everywhere-defined.

Most of our results would also be valid in a more general setting; for instance we could relax the $\mathrm{C}^{1}$ condition on $J$, but this is not a major matter for this paper. There are also some specific other assumptions that we shall make in Section 5 .

2.1. Viscosity solutions of the equation. Equations such as (1.1) can be treated by various ways, depending on the coefficients $a(\cdot)$ and $b(\cdot)$ and whether the measure $\mu$ is singular at the origin or not. Since we make only general assumptions, the best tool in order to deal with differential terms and a singular measure is provided by the notion of viscosity solutions.

Essentially, the notion of a viscosity solution allows us to give sense to the differential terms as well as the integral term without knowing a priori that the solution is regular, using comparison with smooth test functions. Notice however that in the 
integral term, one only needs to replace $u$ by a smooth function in a small neighborhood of $y=0$. We refer to [8, 15, 16, for more results on viscosity solutions in the presence of singular measures. So, if we set

$$
\begin{aligned}
\mathrm{Eq} & {[u, \phi, \delta]\left(x_{0}, t_{0}\right):=\frac{\partial \phi}{\partial t}\left(x_{0}, t_{0}\right)-\operatorname{Tr}\left(a(x) D^{2} \phi\left(x_{0}, t_{0}\right)\right)-b\left(x_{0}\right) \cdot D \phi\left(x_{0}, t_{0}\right) } \\
& -\int_{\{|y|<\delta\}}\left\{\left(\phi\left(x_{0}+y, t_{0}\right)-\phi\left(x_{0}, t_{0}\right)-\left(D \phi\left(x_{0}, t_{0}\right) \cdot y\right)\right\} J(y) \mathrm{d} y\right. \\
& -\int_{\{|y| \geqslant \delta\}}\left\{\left(u\left(x_{0}+y, t_{0}\right)-u\left(x_{0}, t_{0}\right)-\left(D \phi\left(x_{0}, t_{0}\right) \cdot y\right) \mathbb{I}_{\{|y|<1\}}\right\} J(y) \mathrm{d} y\right.
\end{aligned}
$$

we then introduce the following definition:

Definition 2.1. A locally bounded u.s.c. function $u: \mathbb{R}^{N} \times[0, \infty) \rightarrow \mathbb{R}$ is a subsolution of (1.1) iff, for any $\delta \in(0,1)$ and any test function $\phi \in C^{2}\left(\mathbb{R}^{N} \times \mathbb{R}_{+}\right)$, at each maximum point $\left(x_{0}, t_{0}\right) \in \mathbb{R}^{N} \times \mathbb{R}_{+}$of $u-\phi$, we have

$$
\operatorname{Eq}[u, \phi, \delta]\left(x_{0}, t_{0}\right) \leqslant 0
$$

A locally bounded l.s.c. viscosity supersolution is defined in the same way with reversed inequality and min instead of max. Finally, a viscosity solution is a function for which the upper and lower semicontinuous envelopes are respectively a sub- and supersolution of the problem.

When initial and/or boundary data are involved, the definition takes into account that at a boundary point, either the inequation has to hold, or the boundary data has to be taken in the sub/super solution sense. If $\Omega$ is the domain of definition, $f$ is the boundary data (in the sense of nonlocal equations) and $u(x, 0)=u_{0}(x)$ is the initial data of the problem we define:

Definition 2.2. A locally bounded u.s.c. function $u: \mathbb{R}^{N} \times[0, \infty) \rightarrow \mathbb{R}$ is a subsolution of (1.1) with boundary data $u=f$ in $\left(\mathbb{R}^{N} \backslash \Omega\right) \times(0, \infty)$ and initial data $u(x, 0)=u_{0}(x)$ iff, for any $\delta>0$ and any test function $\phi \in C^{2}\left(\mathbb{R}^{N} \times(0, \infty)\right)$, at each maximum point $\left(x_{0}, t_{0}\right) \in \Omega \times[0, \infty)$ of $u-\phi$, we have

$$
\begin{aligned}
\left(x_{0}, t_{0}\right) \in \Omega \times(0, \infty) & \Rightarrow \operatorname{Eq}[u, \phi, \delta]\left(x_{0}, t_{0}\right) \leqslant 0, \\
\left(x_{0}, t_{0}\right) \in \partial \Omega \times(0, \infty) & \Rightarrow \min \left(\operatorname{Eq}[u, \phi, \delta]\left(x_{0}, t_{0}\right) ; u\left(x_{0}, t_{0}\right)-f\left(x_{0}, t_{0}\right)\right) \leqslant 0, \\
\left(x_{0}, t_{0}\right) \in \Omega \times\{0\} & \Rightarrow u\left(x_{0}, t_{0}\right) \leqslant u_{0}\left(x_{0}\right) .
\end{aligned}
$$

A supersolution is defined with reversed inequalities and $\mathrm{min} / \mathrm{max}$ changed accordingly, and a solution is such that its l.s.c./u.s.c. envelopes are sub/super solutions of the equation.

2.2. Hamiltonians. We shall now show some properties of the specific Hamiltonians we consider:

Lemma 2.3. Let $J$ be a kernel satisfying (2.1). Then the Hamiltonian $H$ is superlinear and strictly convex, and thus the associated Lagrangian $L$ is well-defined, convex and superlinear. 
Proof. A straightforward calculus shows that, in the sense of matrices,

$$
D^{2} H(p)=a_{\infty}+\int_{\mathbb{R}^{N}}(y \otimes y) \mathrm{e}^{p \cdot y} J(y) \mathrm{d} y>0
$$

so that $H$ is strictly convex. Now, one can easily check that

$$
H(p)=O\left(|p|^{3}\right)+\int_{\{|y|>1\}} \mathrm{e}^{p \cdot y} J(y) \mathrm{d} y .
$$

Indeed, the differential terms are clearly of the order of $|p|^{2}$ and $|p|$ respectively, while the integral over $|y|<1$ can be bounded by:

$$
\int_{\{|y|<1\}}\left(\mathrm{e}^{p \cdot y}-1-(p \cdot y)\right) J(y) \mathrm{d} y \leqslant C|p|^{2} \int_{\{|y|<1\}} \frac{|y|^{2}}{2}(1+O(p \cdot y)) J(y) \mathrm{d} y \leqslant C|p|^{3} .
$$

On the other hand,

$$
\int_{\{|y|>1\}} \mathrm{e}^{p \cdot y} J(y) \mathrm{d} y \geqslant \int_{\left\{p \cdot y>\rho_{0}|p| / 2\right\}} \mathrm{e}^{p \cdot y} J(y) \mathrm{d} y \geqslant c(J) \mathrm{e}^{\rho_{0}|p| / 2},
$$

so that indeed $H$ is superlinear. It is well known (see [36] for instance) that if $H$ is strictly convex and superlinear, so is $L$.

We shall go a bit further in this direction, using that the main contribution of the Hamiltonian comes from the exponential term in the integral. So let us define the essential part of the Hamiltonian and the corresponding Lagrangian:

Definition 2.4. We denote by

$$
H^{\text {ess }}(p):=\int_{\left\{|y|>\rho_{0} / 2\right\}} \mathrm{e}^{p \cdot y} J(y) \mathrm{d} y \quad \text { and } \quad L^{\mathrm{ess}}(q):=\sup _{p \in \mathbb{R}^{N}}\left\{p \cdot q-H^{\mathrm{ess}}(p)\right\},
$$

where $\rho_{0}$ is defined in (2.1).

The reason why we integrate over $\left\{|y|>\rho_{0} / 2\right\}$ is that we want to avoid the singularity at the origin, but we also want to be sure to integrate within the support of $J$ when it is compactly supported. The condition $\operatorname{supp}(J) \supset B_{\rho_{0}}$ ensures that $\left\{|y|>\rho_{0} / 2\right\} \cap \operatorname{supp}(J) \neq \emptyset$.

First we have a basic estimate, similar to the one that was used in Lemma 2.3 .

Lemma 2.5. Let $J$ be a kernel satisfying (2.1) and (2.5). Then we have

$$
\begin{aligned}
H^{\mathrm{ess}}(p) & \geqslant c(J) \mathrm{e}^{\rho_{0}|p| / 2}, \\
p \cdot D H^{\operatorname{ess}}(p) & \geqslant c(J)\left(\rho_{0}|p| / 2\right) \mathrm{e}^{\rho_{0}|p| / 2}+O(|p|) .
\end{aligned}
$$

Moreover there exists $\varepsilon>0$ such that for any unit vector $\nu$,

$$
\nu^{T} D^{2} H^{\mathrm{ess}}(p) \nu \geqslant \varepsilon c(J) \mathrm{e}^{|p| \rho_{0} / 2}
$$

and the same result holds for $D^{2} H=a_{\infty}+D^{2} H^{\text {ess }} \geqslant D^{2} H^{\text {ess }}$.

Proof. The first estimate is easily obtained:

$$
\begin{aligned}
H^{\text {ess }}(p) & \geqslant \int_{\left\{p \cdot y>\rho_{0}|p| / 2\right\}} \mathrm{e}^{p \cdot y} J(y) \mathrm{d} y \\
& \geqslant c(J) \mathrm{e}^{\rho_{0}|p| / 2} .
\end{aligned}
$$

Notice that the constant $c(J)$ may be small, but it is still positive since $J$ is continuous and positive inside $\left\{p \cdot y>\rho_{0}|p| / 2\right\}$. 
For the second estimate, the proof is essentially the same:

$$
\begin{aligned}
p \cdot D H^{\mathrm{ess}}(p) & \geqslant \int_{\left\{p \cdot y>\rho_{0}|p| / 2\right\}}(p \cdot y) \mathrm{e}^{p \cdot y} J(y) \mathrm{d} y+\int_{\{p \cdot y<0\}}(p \cdot y) \mathrm{e}^{p \cdot y} J(y) \mathrm{d} y \\
& \geqslant c(J)\left(\rho_{0}|p| / 2\right) \mathrm{e}^{\rho_{0}|p| / 2}+O(|p|)-c(J)|p|,
\end{aligned}
$$

where in this case $c(J)=\int_{\{|y|>1\}}|y| J(y) \mathrm{d} y<\infty$ from (2.5).

Finally, for any unit vector $e_{i}$ of the canonical basis of $\mathbb{R}^{N}$, we compute with the same decomposition:

$$
e_{i}^{T} D^{2} H^{\mathrm{ess}}(p) e_{i}=\int_{\left\{|y|>\rho_{0} / 2\right\}}\left|y_{i}\right|^{2} \mathrm{e}^{p \cdot y} J(y) \mathrm{d} y \geqslant \int_{\left\{p \cdot y>\rho_{0}|p| / 2\right\}}\left|y_{i}\right|^{2} \mathrm{e}^{p \cdot y} J(y) \mathrm{d} y .
$$

We notice that the set $\left\{p \cdot y>\rho_{0}|p| / 2\right\}$ contains the truncated cone:

$$
\left\{\frac{p \cdot y}{|p||y|} \geqslant \frac{3}{4},|y|>\frac{3 \rho_{0}}{4}\right\}
$$

hence for some $\varepsilon>0$ small enough (which can be chosen independently of $e_{i}$ ),

$$
\mathcal{B}:=\left\{p \cdot y>\rho_{0}|p| / 2\right\} \cap\left\{\left|y_{i}\right|^{2} \geqslant \varepsilon\right\} \neq \emptyset,
$$

and since $\mathcal{B} \cap B_{\rho_{0}} \neq \emptyset$, the integral of $J$ on $\mathcal{B}$ is positive. So we can estimate the integral from below as follows:

$$
\begin{aligned}
e_{i}^{T} D^{2} H^{\mathrm{ess}}(p) e_{i} & \geqslant \varepsilon \mathrm{e}^{|p| \rho_{0} / 2} \int_{\mathcal{B}} J(y) \mathrm{d} y \\
& \geqslant \varepsilon c(J) \mathrm{e}^{|p| \rho_{0} / 2} .
\end{aligned}
$$

So the result also holds for any unit vector $\nu$.

The next estimate will be essential in the sequel:

Lemma 2.6. Let $J$ be a kernel satisfying (2.1) and (2.4). Then, for any $\gamma \in\left(0, \rho_{0}\right)$,

$$
H^{\mathrm{ess}}(p) \leqslant \frac{c(J)}{\gamma}+\frac{p \cdot D H^{\mathrm{ess}}(p)}{\gamma|p|}+\mathrm{e}^{\gamma|p|} .
$$

As a consequence, $H^{\mathrm{ess}}(p)$ is negligible in front of $p \cdot D H^{\mathrm{ess}}(p)$ as $|p| \rightarrow \infty$,

$$
\lim _{|p| \rightarrow \infty} \frac{H^{\mathrm{ess}}(p)}{p \cdot D H^{\mathrm{ess}}(p)}=0
$$

Proof. We begin with splitting the integral into two terms,

$$
\begin{aligned}
H^{\text {ess }}(p) & =\int_{\{p \cdot y<\gamma|p|\} \cap\left\{|y| \geqslant \rho_{0} / 2\right\}} \mathrm{e}^{p \cdot y} J(y) \mathrm{d} y+\int_{\{p \cdot y \geqslant \gamma|p|\} \cap\left\{|y| \geqslant \rho_{0} / 2\right\}} \mathrm{e}^{p \cdot y} J(y) \mathrm{d} y \\
& =I_{1}+I_{2} .
\end{aligned}
$$

The first integral is estimated as follows:

$$
I_{1} \leqslant \int_{\{p \cdot y<\gamma|p|\} \cap\left\{|y| \geqslant \rho_{0} / 2\right\}} \mathrm{e}^{\gamma|p|} J(y) \mathrm{d} y \leqslant c(J) \mathrm{e}^{\gamma|p|} .
$$

Now, notice that since $\gamma<\rho_{0}$, even if $J$ is compactly supported, we are sure that the integral $I_{2}$ concerns a region where $J$ is not zero, so that we can indeed control 
it by $p \cdot D H^{\operatorname{ess}}(p)$ :

$$
\begin{aligned}
I_{2} & \leqslant \frac{1}{\gamma|p|} \int_{\{p \cdot y \geqslant \gamma|p|\}}(p \cdot y) \mathrm{e}^{p \cdot y} J(y) \mathrm{d} y \\
& =\frac{1}{\gamma|p|} \int_{\mathbb{R}^{N}}(p \cdot y) \mathrm{e}^{p \cdot y} J(y) \mathrm{d} y-\frac{1}{\gamma|p|} \int_{\{p \cdot y \leqslant \gamma|p|\}}(p \cdot y) \mathrm{e}^{p \cdot y} J(y) \mathrm{d} y \\
& \leqslant \frac{p \cdot D H^{\operatorname{ess}}(p)}{\gamma|p|}-\frac{1}{\gamma|p|} \int_{\{p \cdot y \leqslant 0\}}(p \cdot y) \mathrm{e}^{p \cdot y} J(y) \mathrm{d} y \\
& \quad-\frac{1}{\gamma|p|} \int_{\{0 \leqslant p \cdot y \leqslant \gamma|p|\}}(p \cdot y) \mathrm{e}^{p \cdot y} J(y) \mathrm{d} y \\
& \leqslant \frac{p \cdot D H^{\operatorname{ess}}(p)}{\gamma|p|}+\frac{1}{\gamma|p|} \int_{\{p \cdot y \leqslant 0\}}|p||y| J(y) \mathrm{d} y \\
& \leqslant \frac{p \cdot D H^{\operatorname{ess}}(p)}{\gamma|p|}+\frac{c(J)}{\gamma} .
\end{aligned}
$$

Summing up $I_{1}$ and $I_{2}$ gives the first result of the lemma.

For the second part we first fix $\mu>1, \gamma=\mu /|p|$ and choose $|p|>\mu / \rho_{0}$ so that $\gamma \in\left(0, \rho_{0}\right)$. Using estimate (2.6), we obtain:

$$
H^{\mathrm{ess}}(p) \leqslant \frac{c(J)|p|}{\mu}+\frac{p \cdot D H^{\mathrm{ess}}(p)}{\mu}+\mathrm{e}^{\mu},
$$

which implies

$$
\frac{p \cdot D H^{\mathrm{ess}}(p)}{H^{\mathrm{ess}}(p)} \geqslant \mu-\frac{c(J)|p|}{H^{\mathrm{ess}}(p)}-\frac{\mu \mathrm{e}^{\mu}}{H^{\mathrm{ess}}(p)} .
$$

Since $H^{\text {ess }}(p) \rightarrow \infty$ superlinearly (see Lemma 2.5) we then have

$$
\liminf _{|p| \rightarrow \infty} \frac{p \cdot D H^{\mathrm{ess}}(p)}{H^{\mathrm{ess}}(p)} \geqslant \mu
$$

But since $\mu>1$ is arbitrary, we conclude that the limit is $+\infty$, hence the result.

Remark 2.7. If $D H^{\text {ess }}(p)$ would grow faster than an exponential, estimate (2.6) would be sufficient to conclude that $H^{\mathrm{ess}}(p)$ is negligible in front of $p \cdot D H^{\mathrm{ess}}(p)$. Actually this will be the case when $J$ is not compactly supported; see Lemma 5.7

The following lemma proves that, essentially, the estimates we will produce in this paper do not depend on $A, B$ and neither on the behaviour of $J$ near the origin.

Lemma 2.8. For any J satisfying (2.1) and (2.4), as $|p| \rightarrow \infty$, we have

$$
H(p) \sim H^{\mathrm{ess}}(p), D H(p) \sim D H^{\mathrm{ess}}(p) \text { and } L(p) \sim L^{\mathrm{ess}}(p) .
$$

Moreover, if $J$ is symmetric, then $H^{\mathrm{ess}}$ and $L^{\mathrm{ess}}$ are also symmetric. 
Proof. We split the Hamiltonian as follows:

$$
\begin{aligned}
H(p) & =\operatorname{Tr}\left(a_{\infty} p \otimes p\right)+b_{\infty} \cdot p+H^{\mathrm{ess}}(p)+\int_{\left\{|y| \leqslant \rho_{0} / 2\right\}}\left(\mathrm{e}^{p \cdot y}-1-(p \cdot y)\right) J(y) \mathrm{d} y \\
& -\int_{\left\{\rho_{0} / 2<|y| \leqslant 1 \mid\right\}}(1+(p \cdot y)) J(y) \mathrm{d} y-\int_{\{|y|>1\}} J(y) \mathrm{d} y \\
& =H^{\operatorname{ess}}(p)+\operatorname{Tr}\left(a_{\infty} p \otimes p\right)+b_{\infty} \cdot p \\
& +\int_{\left\{|y| \leqslant \rho_{0} / 2\right\}}\left(\frac{(p \cdot y)^{2}}{2}(1+O(p \cdot y))\right) J(y) \mathrm{d} y+c(J) \\
& =H^{\operatorname{ess}}(p)+O\left(|p|^{3}\right) .
\end{aligned}
$$

Since the Hamiltonians behave at least exponentially, Lemma 2.5. the $O\left(|p|^{3}\right)$ is negligible by far, and $H$ and $H^{\text {ess }}$ are equivalent. The calculations for $D H$ and $D H^{\text {ess }}$ are similar: we get

$$
\left|D H(p)-D H^{\mathrm{ess}}(p)\right|=O\left(|p|^{2}\right),
$$

while $|D H(p)|$ grows at least exponentially. Notice that since $H$ and $H^{\text {ess }}$ are equivalent, Lemma 2.6 implies that both are negligible in front of $D H$ and $D H^{\text {ess }}$.

More involved is the proof that $L^{\mathrm{ess}}(p) \sim L(p)$. Let us notice first that the point $p_{0}=p_{0}(q)$ where $L(q)$ reaches its sup goes to infinity as $|q| \rightarrow \infty$. Indeed, this comes from the fact that $q=D H\left(p_{0}\right)$ and that $D H(p)$ grows at least exponentially; see Lemma 2.5. The same holds for $D H^{\text {ess }}$.

Then we proceed as follows: for $|q|$ large enough, since the sup below are attained for $|p|$ also large, there is some constant $C>0$ such that

$$
\sup _{p \in \mathbb{R}^{N}}\left\{p \cdot y-H^{\text {ess }}(p)-C|p|^{3}\right\} \leqslant L(q) \leqslant \sup _{p \in \mathbb{R}^{N}}\left\{p \cdot y-H^{\text {ess }}(p)+C|p|^{3}\right\} .
$$

Hence if we denote by $p_{1}=p_{1}(q)$ the point where the sup on the right is attained, we have $q=D H^{\text {ess }}\left(p_{1}\right)+3 C\left|p_{1}\right| p_{1}$, while $q=D H\left(p_{0}\right)$ for the sup in $L(q)$. Now,

$q=D H^{\mathrm{ess}}\left(p_{1}\right)+3 C\left|p_{1}\right| p_{1}=D H^{\mathrm{ess}}\left(p_{1}\right)+O\left(\left|p_{1}\right|^{2}\right)=D H\left(p_{1}\right)+O\left(\left|p_{1}\right|^{2}\right)=D H\left(p_{0}\right)$, and from this we will conclude that $p_{1}(q) \sim p_{0}(q)$. More precisely,

$$
O\left(\left|p_{1}\right|^{2}\right)=D H\left(p_{1}\right)-D H\left(p_{0}\right)=D^{2} H(\xi) \cdot\left(p_{1}-p_{0}\right)
$$

for some $\xi$ in the segment $\left[p_{0}, p_{1}\right]$. We take the scalar product with $\left(p_{1}-p_{0}\right)$ and use Lemma 2.5 to get an estimate from above:

$$
\left|p_{1}-p_{0}\right|^{2}\left(\varepsilon c(J) \mathrm{e}^{|\xi| \rho_{0} / 2}\right) \leqslant\left(D^{2} H(\xi) \cdot\left(p_{1}-p_{0}\right) \cdot\left(p_{1}-p_{0}\right)\right) \leqslant C\left|p_{1}\right|^{2}\left|p_{1}-p_{0}\right| .
$$

From this it follows that for some constant still noted $C>0$,

$$
\left|p_{1}-p_{0}\right| \leqslant C \frac{\mathrm{e}^{-|\xi| \rho_{0} / 2}}{\left|p_{1}\right|^{2}}
$$

Noticing that $|\xi| \geqslant \min \left\{\left|p_{0}\right|,\left|p_{1}\right|\right\} \rightarrow \infty$, this estimate implies that as $|q| \rightarrow \infty$,

$$
\frac{\left|p_{1}-p_{0}\right|}{\min \left\{\left|p_{0}\right|,\left|p_{1}\right|\right\}} \leqslant \frac{C}{\left|p_{1}\right|^{2}} \frac{\mathrm{e}^{-\min \left\{\left|p_{0}\right|,\left|p_{1}\right|\right\} \rho_{0} / 2}}{\min \left\{\left|p_{0}\right|,\left|p_{1}\right|\right\}} \rightarrow 0 .
$$

Hence we have proved that $p_{1}(q) \sim p_{0}(q)$. 
The same calculation is valid for the sup on the left of (2.7), so that we conclude that

$$
L^{\mathrm{ess}}(q)=p_{1} \cdot D H^{\mathrm{ess}}\left(p_{1}\right)-H^{\mathrm{ess}}\left(p_{1}\right) \sim p_{0} \cdot D H\left(p_{0}\right)-H\left(p_{0}\right)=L(q) .
$$

About the symmetry, see [12, Lemma 2.4].

We finally prove a result which allows us to compare the Lagrangians when the kernels are ordered. But for some technical reasons, we need the kernels to be strictly ordered in $B_{\rho} \backslash B_{\rho_{0} / 2}$; hence we introduce the following notation:

Definition 2.9. We say that $J_{1}$ and $J_{2}$ are essentially ordered and we denote $J_{1} \prec J_{2}$ if there exist $a, b>0$ such that $\rho_{0} / 2<a<b<\rho_{0}$ and

$$
J_{1} \leqslant J_{2} \text { in } \mathbb{R}^{N}, \quad J_{1}(y)<J_{2}(y) \text { in }\{a<|y|<b \mid\} .
$$

Lemma 2.10. Let us assume that $J_{1}, J_{2}$ satisfy (2.1) and (2.4) and that $J_{1} \prec J_{2}$. Denote by $H_{1}, H_{2}, L_{1}, L_{2}$ the associated Hamiltonians and Lagrangians. Then for $p, q$ big enough:

$$
H_{1}(p) \leqslant H_{2}(p) \quad \text { and } \quad L_{1}(q) \geqslant L_{2}(q) .
$$

Proof. The inequality $H_{1}^{\text {ess }}(p) \leqslant H_{2}^{\text {ess }}(p)$ is always true, since the integrand in $H^{\text {ess }}$ is always positive and $J_{1} \leqslant J_{2}$. But as such, this is not enough to derive the same inequality between $H_{1}$ and $H_{2}$. To this end, let us notice that since $J_{1} \prec J_{2}$, then there exists $\varepsilon>0$ and $\rho_{0} / 2<a<b<\rho_{0}$ such that

$$
J_{1}(y) \leqslant J_{2}(y)-\varepsilon \chi(y) \text { in } \mathbb{R}^{N},
$$

where $\chi(x)=((b-|x|)(|x|-a))_{+}$.

Then we shall estimate the difference $H_{1}^{\mathrm{ess}}-H_{2}^{\mathrm{ess}}$ as in Lemma 2.5

$$
\begin{aligned}
H_{1}^{\text {ess }}(p)-H_{2}^{\text {ess }}(p) & \leqslant-\varepsilon \int_{\{a<|y|<b\}} \mathrm{e}^{p \cdot y} \chi(y) \mathrm{d} y \\
& \leqslant-\varepsilon \int_{\{a<|y|<b\} \cap\{p \cdot y>a|p|\}} \mathrm{e}^{p \cdot y} \chi(y) \mathrm{d} y \\
& \leqslant-\varepsilon c(\chi) \mathrm{e}^{a|p|},
\end{aligned}
$$

where $c(\chi)$ is the integral of $\chi$ over $\{a<|y|<b\} \cap\{p \cdot y>a|p|\}$. It is clear that this set has nonempty interior and since $\chi>0$ in this set, then $c(\chi)$ is a strictly positive constant. This proves that not only are $H_{1}^{\text {ess }}$ and $H_{2}^{\text {ess }}$ ordered, but moreover the difference between the two is at least of the order of an exponential.

Then we use, as in the proof of Lemma 2.8, that $H_{1}(p)=H_{1}^{\mathrm{ess}}(p)+O\left(|p|^{3}\right)$ and the same for $\mathrm{H}_{2}$, so that

$$
H_{1}(p)-H_{2}(p)=H_{1}^{\mathrm{ess}}(p)-H_{2}^{\mathrm{ess}}(p)+O\left(|p|^{3}\right) \leqslant-\varepsilon c(\chi) \mathrm{e}^{a|p|}+O\left(|p|^{3}\right) .
$$

Thus for $|p|$ big enough, we have indeed $H_{1}(p) \leqslant H_{2}(p)$.

The inequality for $L_{1}$ and $L_{2}$ follows by definition.

Remark 2.11. ( $i$ ) This last result is also valid if $J_{1}$ satisfies (2.5) with $\beta_{1}, J_{2}$ satisfies (2.5) with $\beta_{2} \leqslant \beta_{1}$ and we consider $|p|<\beta_{2}$. (ii) Notice that if we only assume $J_{1}=J_{2}$ outside $B_{\rho}$ and $J_{1}<J_{2}$ in $B_{\rho}$, then $J_{1} \prec J_{2}$. In this case, the tails of both kernels and their support (if compactly supported) remain exactly the same so that, essentially, all the estimates we give in the sequel are the same for both kernels. 
2.3. Viscosity solutions of the limit Hamilton-Jacobi equation. In Section 3, we will have to study the following Hamilton-Jacobi equation with CauchyDirichlet boundary values:

$$
\begin{cases}\partial_{t} I^{A}+H\left(D I^{A}\right)=0 & \text { in } B_{1} \times(0, \infty), \\ I^{A}=0 & \text { on } \partial B_{1} \times(0, \infty), \\ I^{A}(x, 0)=A & \text { in } B_{1},\end{cases}
$$

where we assume here that $H \in \mathrm{C}\left(\mathbb{R}^{N} ; \mathbb{R}\right)$. We refer to Section 6 for the case when the Hamiltonian could take infinite values. Let us first recall the definition of viscosity solutions for this equation (see for instance [23]):

Definition 2.12. A locally bounded u.s.c. function $u: \bar{B}_{1} \times[0, \infty) \rightarrow \mathbb{R}$ is a viscosity sub-solution of (2.9) if for any $\mathrm{C}^{2}$-smooth function $\varphi$, and any point $\left(x_{0}, t_{0}\right) \in \bar{B}_{1} \times[0, \infty)$ where $u-\varphi$ reaches a maximum, it follows that

$$
\begin{aligned}
x_{0} \in B_{1} & \Rightarrow \partial_{t} \varphi+H(D \varphi) \leqslant 0 \text { at }\left(x_{0}, t_{0}\right), \\
x_{0} \in \partial B_{1} & \Rightarrow \min \left\{\partial_{t} \varphi+H(D \varphi) ; u\right\} \leqslant 0 \text { at }\left(x_{0}, t_{0}\right), \\
t_{0}=0 & \Rightarrow u \leqslant A \text { in } B_{1} .
\end{aligned}
$$

A locally bounded l.s.c. function is a viscosity super-solution if the same holds with reversed inequalities and min replaced by max at the boundary. Finally a viscosity solution is a locally bounded function $u$ such that its u.s.c. and l.s.c. envelopes are respectively sub- and super-solutions of (2.9).

Notice that, in general, the initial data has to be understood in the relaxed viscosity way (either the data is taken, or the sub/super-solution condition holds), but it is well known (see for instance [3]) that in the case of a continuous Hamiltonian, this condition is equivalent to the one we use here. Keep in mind, however, that this will not be the case in Section 6 .

Since $H$ is convex we have the following representation:

Lemma 2.13. If $J$ satisfies (2.1) and (2.4), then there exists a unique viscosity solution of (2.9), which is given by

$$
I^{A}(x, t)=A \wedge \min _{y \in \partial B_{1}}\left\{t L\left(\frac{x-y}{t}\right)\right\} \quad \text { in } \quad B_{1} \times(0, \infty) .
$$

Proof. The assumptions made on $J$ imply that both $H$ and $L$ are finite everywhere, convex and super-linear so that uniqueness holds for this problem; see [33]. Then the Lax-Oleinik formula in the bounded domain $B_{1} \times(0, \infty)$ gives:

$$
I^{A}(x, t)=\min _{|y| \leqslant 1}\left\{t L\left(\frac{y-x}{t}\right)+A\right\} \wedge \min _{(y, s) \in \partial B_{1} \times(0, t)}\left\{(t-s) L\left(\frac{x-y}{t-s}\right)\right\} .
$$

Using that $L \geqslant 0$ and $L(0)=0$, we obtain that the first min equals $A$. Then using that the function $r \mapsto L(c r) / r$ is increasing, the second minimum is attained for $s=0$ so that the result holds.

\section{Theoretical Behaviour}

The main goal of this section is to derive a theoretical bound, in terms of the Lagrangian $L$, for the error made when approximating the solution, $u$, of (1.1) with initial data $u_{0}$ by solutions $u_{R}$ of (1.3). This result will allow us to derive explicit rates of convergence provided we know the behaviour at infinity of $L(q)$ (this will be the aim of the next sections). 
Theorem 3.1. If $J$ satisfies (2.1) and (2.4), then for any fixed $x \in \mathbb{R}^{N}$ and $t>0$, as $R \rightarrow \infty$, it follows that

$$
\left|u-u_{R}\right|(x, t) \leqslant \mathrm{e}^{-R I_{\infty}(x / R, t / R)+o(1) R},
$$

where o(1) are terms that tend to zero as $R$ tends to $\infty$. The rate function is given by

$$
I_{\infty}(x, t)=\min _{y \in \partial B_{1}} t L\left(\frac{x-y}{t}\right) .
$$

and, moreover, for any $T>0$ the o(1) is uniform in sets of the form

$$
\{|x / R| \leqslant 1,0 \leqslant t / R \leqslant T\} .
$$

We shall dedicate the rest of this section to the proof of this theorem; first we do it formally, so that the main structure of the proof will be easier to follow and then deal with all the technical details. Notice that if one only assumes (2.5) instead of (2.4), the theorem still remains valid, but the proof requires some finer arguments since the Hamiltonian can be infinite in some regions of $\mathbb{R}^{N}$. In such a case, an initial layer appears that we analyze in detail in Section 6 .

3.1. Formal proof of Theorem 3.1. Let us denote by $v_{R}$ the solution of (1.1) in $B_{R} \times(0, \infty)$ with initial value $v_{R}(x, 0)=0$ for $x \in B_{R}$ and "boundary data" $v_{R}=\left\|u_{0}\right\|_{\infty}$ for $|x| \geqslant R$. By comparison, $0 \leqslant u-u_{R} \leqslant v_{R}$ so that we need only to estimate $v_{R}$.

We first rescale the equation both in $x$ and $t$ as follows:

$$
w_{R}(x, t)=v_{R}(R x, R t) \quad \text { for } \quad x \in B_{1}, t \geqslant 0 .
$$

Then $w_{R}$ satisfies a rescaled equation in the fixed ball $B_{1}$ in the sense of viscosity:

$$
\begin{aligned}
\partial_{t} w_{R}(x, t) & =\frac{1}{R} \operatorname{Tr}\left(a(R x) D^{2} w_{R}(x, t)\right)+b(R x) \cdot D w_{R}(x, t) \\
& +R \int_{\mathbb{R}^{N}}\left\{w_{R}(x+y / R, t)-w_{R}(x, t)-\left(D w_{R}(x) \cdot y / R\right) \mathbb{I}_{\{|y|<1\}}(y)\right\} J(y) \mathrm{d} y .
\end{aligned}
$$

In order to estimate $w_{R}$ we follow [6] and perform the "usual" logarithmic transform, but we have to rescale accordingly, dividing by $R$ (and not $R^{2}$ as is the case for the heat equation). So, remembering that for $t>0, w_{R}>0$, let us define

$$
I_{R}(x, t)=-\frac{1}{R} \ln \left(w_{R}(x, t)\right) .
$$

Then formally as $R \rightarrow \infty$, setting $I=\lim I_{R}$ we have

$$
\begin{array}{ll}
I(x, t)=0, & (x, t) \in \partial B_{1} \times(0, \infty), \\
I(x, 0)=\infty, & x \in B_{1} .
\end{array}
$$

In order to find an expression for the equation satisfied by $I_{R}$ we compute

$$
\begin{aligned}
& \partial_{t} w_{R}(x, t)=-R \mathrm{e}^{-R I_{R}(x, t)} \partial_{t} I_{R}(x, t), \quad D w_{R}(x, t)=-R \mathrm{e}^{-R I_{R}(x, t)} D I_{R}(x, t), \\
& D^{2} w_{R}(x, t)=-R \mathrm{e}^{-R I_{R}(x, t)} D^{2} I_{R}(x, t)+R^{2} \mathrm{e}^{-R I_{R}(x, t)}\left(D I_{R} \otimes D I_{R}\right)(x, t),
\end{aligned}
$$


and

$$
\begin{aligned}
\int_{\mathbb{R}^{N}} & \left\{w_{R}(x+y / R, t)-w_{R}(x, t)-\left(D w_{R}(x) \cdot y / R\right) \mathbf{I}_{\{|y|<1\}}(y)\right\} J(y) \mathrm{d} y \\
= & \int_{\mathbb{R}^{N}}\left\{\mathrm{e}^{-R I_{R}(x+y / R, t)}-\mathrm{e}^{-R I_{R}(x, t)}+\left(D I_{R}(x) \cdot y\right) \mathrm{e}^{-R I_{R}(x, t)} \mathbf{I}_{\{|y|<1\}}(y)\right\} J(y) \mathrm{d} y \\
= & \mathrm{e}^{-R I_{R}(x, t)} \int_{\mathbb{R}^{N}}\left\{\mathrm{e}^{R\left\{-I_{R}(x+y / R, t)+I_{R}(x, t)\right\}}-1+\left(D I_{R}(x) \cdot y\right) \mathbb{I}_{\{|y|<1\}}(y)\right\} J(y) \mathrm{d} y .
\end{aligned}
$$

As for the differential terms, we have

$$
\begin{aligned}
& -\mathrm{e}^{R I_{R}(x)}\left(\frac{1}{R^{2}} \operatorname{Tr}\left(a(R x) D^{2} w_{R}(x, t)\right)+\frac{1}{R} b(R x) \cdot D w_{R}(x, t)\right) \\
& \quad=\frac{1}{R} \operatorname{Tr}\left(a(R x) D^{2} I_{R}(x, t)\right)-\operatorname{Tr}\left(a(R x) D I_{R}(x, t) \otimes D I_{R}(x, t)\right)-b(R x) \cdot D I_{R}(x, t) .
\end{aligned}
$$

We thus arrive at the following equation for $I_{R}$ :

$$
\begin{aligned}
& \partial_{t} I_{R}(x, t)+\int_{\mathbb{R}^{N}}\left\{\mathrm{e}^{-R\left\{I_{R}(x+y / R, t)-I_{R}(x, t)\right\}}-1+\left(D I_{R}(x) \cdot y\right) \mathbb{I}_{\{|y|<1\}}(y)\right\} J(y) \mathrm{d} y \\
& =\frac{1}{R} \operatorname{Tr}\left(a(R x) D^{2} I_{R}(x, t)\right)-\operatorname{Tr}\left(a(R x) D I_{R}(x, t) \otimes D I_{R}(x, t)\right)-b(R x) \cdot D I_{R}(x, t) .
\end{aligned}
$$

Using the limits defined in (2.3), the equation formally converges to the HamiltonJacobi equation

$$
\partial_{t} I+H(D I)=0
$$

with

$$
H(p)=\operatorname{Tr}\left(a_{\infty} p \otimes p\right)+b_{\infty} \cdot p+\int\left(\mathrm{e}^{p \cdot y}-1-(p \cdot y) \mathbf{I}_{\{|y|<1\}}\right) J(y) \mathrm{d} y .
$$

The main point now is solving the Cauchy-Dirichlet equation given by (3.5) together with the data (3.3), which can be done using the Lax-Oleinik formula, according to Lemma 2.13. We have formally obtained

$$
I(x, t)=\min _{y \in \partial B_{1}}\left\{t L\left(\frac{x-y}{t}\right)\right\} .
$$

In the following subsection we then justify the convergence of $I_{R}$ towards the solution in the sense of viscosity solutions.

Remark 3.2. We see here that if we want to keep track of the nonlocal term, and obtain a nontrivial limit Hamilton-Jacobi equation for the rate function, we need to balance three parameters altogether: the $(1 / R)$ factor coming from the Taylor expansion of $I_{R}(x+y / R, t)-I_{R}(x, t)$, the $R$-factor coming from choosing $I_{R}=(1 / R) \ln w_{R}$, and the time scaling factor $R$ in front of the nonlocal term with the same $(1 / R) \ln w_{R}$ choice. This implies that only the hyperbolic scaling will lead to large deviation analysis in the present situation.

3.2. Proof of Theorem 3.1] Passing to the limit in the viscous sense. A first problem in our formal analysis comes from the fact that if $w_{R}$ approaches zero, then $I_{R}$ may not remain bounded. Hence to avoid upper estimates for $I_{R}$, we use the same trick as in [6], which consists in modifying $I_{R}$ a little bit. For any $A>0$, let

$$
I_{R}^{A}(x, t)=-\frac{1}{R} \ln \left(w_{R}(x, t)+\mathrm{e}^{-R A}\right),
$$


which is bounded from above by $A$. Let us notice that since equation (1.1) is invariant under addition of constants, $I_{R}^{A}$ satisfies the same equation as $I_{R}$, that is, equation (3.4).

Proposition 3.3. The sequence $\left(I_{R}^{A}\right)$ converges locally uniformly in $\bar{B}_{1} \times[0, \infty)$ as $R \rightarrow+\infty$ towards the unique viscosity solution $I^{A}$ of (2.9).

Proof. We introduce the half-relaxed limits, for $x \in \overline{B_{1}}, t \geqslant 0$ :

$$
\bar{I}^{A}(x, t):=\limsup _{R \rightarrow \infty} I_{R}^{A}(x, t)=\limsup _{\substack{\left(x^{\prime}, t^{\prime}\right) \rightarrow(x, t) \\ R \rightarrow \infty}} I_{R}^{A}\left(x^{\prime}, t^{\prime}\right)
$$

and

$$
\underline{I}^{A}(x, t):=\liminf _{R \rightarrow \infty}{ }^{*} I_{R}^{A}(x, t)=\liminf _{\substack{\left.x^{\prime}, t^{\prime}\right) \rightarrow(x, t) \\ R \rightarrow \infty}} I_{R}^{A}\left(x^{\prime}, t^{\prime}\right)
$$

and we shall prove that they are respectively viscosity sub- and super-solutions of the limit problem (2.9). Then a uniqueness result will allow us to conclude the proof.

Let us take $\delta \in(0,1)$ and a test function $\phi$ such that $\bar{I}^{A}-\phi$ has a maximum at $\left(x_{0}, t_{0}\right)$. Up to a standard modification of $\phi$, we can assume that the maximum is strict so that there exist sequences $R_{n} \rightarrow+\infty$ and $\left(x_{n}, t_{n}\right) \rightarrow\left(x_{0}, t_{0}\right)$ such that

$$
I_{R_{n}}^{A}-\phi \text { has a strict maximum at }\left(x_{n}, t_{n}\right) \text {. }
$$

Case 1. The point $\left(x_{0}, t_{0}\right)$ is inside $B_{1} \times(0, \infty)$. Then for $n$ big enough, all the points $\left(x_{n}, t_{n}\right)$ are also inside $B_{1} \times(0, \infty)$, so we may use the equation for $I_{R_{n}}^{A}$ at those points and pass to the limit.

We first write the viscosity inequality for $I_{R}^{A}$ :

$$
\begin{aligned}
& \frac{\partial \phi}{\partial t}\left(x_{n}, t_{n}\right) \leqslant \frac{1}{R_{n}} \operatorname{Tr}\left(a\left(R_{n} x_{n}\right) D^{2} \phi\left(x_{n}, t_{n}\right)\right) \\
& \quad-\operatorname{Tr}\left(a\left(R_{n} x_{n}\right) D \phi\left(x_{n}, t_{n}\right) \otimes D \phi\left(x_{n}, t_{n}\right)\right)-b\left(R_{n} x_{n}\right) \cdot D \phi\left(x_{n}, t_{n}\right) \\
& -\int_{\{|y|<\delta\}}\left\{\mathrm{e}^{-R\left\{\phi\left(x_{n}+y / R, t_{n}\right)-\phi\left(x_{n}, t_{n}\right)\right\}}-1-\left(D \phi\left(x_{n}, t_{n}\right) \cdot y\right)\right\} J(y) \mathrm{d} y \\
& -\int_{\{|y| \geqslant \delta\}}\left\{\mathrm{e}^{-R\left\{I_{R_{n}}^{A}\left(x_{n}+y / R, t_{n}\right)-I_{R_{n}}^{A}\left(x_{n}, t_{n}\right)\right\}}-1\right. \\
& \left.\quad-\left(D \phi\left(x_{n}, t_{n}\right) \cdot y\right) \mathbb{I}_{\{|y|<1\}}\right\} J(y) \mathrm{d} y \\
& =\operatorname{Diff}(n)+\operatorname{Int}_{1}(n, \delta)+\operatorname{Int}_{2}(n, \delta),
\end{aligned}
$$

where $\operatorname{Diff}(n)$ represents the differential terms, $\operatorname{Int}_{1}(n, \delta)$ is the integral over $\{|y|<$ $\delta\}$ and $\operatorname{Int}_{2}(n, \delta)$ is the integral over $\{|y| \geqslant \delta\}$.

Let us first remark that passing to the limit in the differential terms is easy; using (2.3):

$$
\operatorname{Diff}(n) \rightarrow-\operatorname{Tr}\left(a_{\infty} \cdot D \phi\left(x_{0}, t_{0}\right) \otimes D \phi\left(x_{0}, t_{0}\right)\right)-b_{\infty} \cdot D \phi\left(x_{0}, t_{0}\right) .
$$

For $n$ big enough, the first integral term can be controlled by:

$$
\left|\operatorname{Int}_{1}(\delta, n)\right| \leqslant\left\|D^{2} \phi\right\|_{L^{\infty}\left(B_{1}\left(x_{0}, t_{0}\right)\right)} \int_{\{|y|<\delta\}}|y|^{2} J(y) \mathrm{d} y=o_{\delta}(1),
$$

where $o_{\delta}(1)$ represents a quantity that goes to zero as $\delta \rightarrow 0$. 
It remains to pass to the limit in $\mathrm{Int}_{2}$. To this end we use the fact that, since we have a maximum point, for any $z \in \mathbb{R}^{N}$,

$$
I_{R_{n}}^{A}\left(x_{n}+z, t_{n}\right)-I_{R_{n}}^{A}\left(x_{n}, t_{n}\right) \leqslant \phi\left(x_{n}+z, t_{n}\right)-\phi\left(x_{n}, t_{n}\right) .
$$

Then, we fix $\varepsilon>0$, choose some $M>1$ and split Int $_{2}$ into two terms as follows:

$$
\begin{aligned}
\operatorname{Int}_{2}(\delta, n) \leqslant & -\int_{\delta \leqslant|y|<M}\left\{\mathrm{e}^{-R\left\{\phi\left(x_{n}+y / R_{n}, t_{n}\right)-\phi\left(x_{n}, t_{n}\right)\right\}}-1\right. \\
& \left.-\left(D \phi\left(x_{n}, t_{n}\right) \cdot y\right) \mathbb{I}_{\{|y|<1\}}\right\} J(y) \mathrm{d} y \\
& +\left|\int_{|y| \geqslant M}\left\{w_{R_{n}}\left(x_{n}+y / R_{n}, t_{n}\right)-w_{R_{n}}\left(x_{n}, t_{n}\right)\right\} J(y) \mathrm{d} y\right| .
\end{aligned}
$$

Since $w_{R}$ is bounded by $\left\|u_{0}\right\|_{\infty}$, we can choose $M$ big enough so that the second term is less than $\varepsilon$, independently of $n$.

Then we write a Taylor expansion for $\phi$ near point $x_{n}$ : there exists a $\xi_{n} \in B_{M}$ such that

$$
\begin{aligned}
\operatorname{Int}_{2} \leqslant-\int_{\delta \leqslant|y|<M}\{ & \mathrm{e}^{-D \phi\left(x_{n}, t_{n}\right) \cdot y+\frac{1}{R_{n}}\left(D^{2} \phi\left(\xi_{n}\right) y \cdot y\right)}-1 \\
& \left.-\left(D \phi\left(x_{n}, t_{n}\right) \cdot y\right) \mathbb{I}_{\{|y|<1\}}\right\} J(y) \mathrm{d} y+\varepsilon .
\end{aligned}
$$

Since $\xi_{n}$ remains in $B_{M}$ and $\phi$ is smooth we have that $D^{2} \phi\left(\xi_{n}\right)$ remains bounded. Hence, we can pass to the limit as $n \rightarrow+\infty$ :

$\limsup _{n \rightarrow \infty} \operatorname{Int}_{2}(\delta, n) \leqslant-\int_{\delta \leqslant|y|<M}\left\{\mathrm{e}^{-D \phi\left(x_{0}, t_{0}\right) \cdot y}-1-\left(D \phi\left(x_{0}, t_{0}\right) \cdot y\right) \mathbb{I}_{\{|y|<1\}}\right\} J(y) \mathrm{d} y+\varepsilon$.

Summing up the various terms, we obtain that for any $\delta>0$ and any $\varepsilon>0$, there exists $M=M(\varepsilon)>1$ such that

$$
\begin{aligned}
\frac{\partial \phi}{\partial t}\left(x_{0}, t_{0}\right) \leqslant & -\operatorname{Tr}\left(a_{\infty} \cdot D \phi\left(x_{0}, t_{0}\right) \otimes D \phi\left(x_{0}, t_{0}\right)\right)-b_{\infty} \cdot D \phi\left(x_{0}, t_{0}\right) \\
& -\int_{\delta \leqslant|y|<M}\left\{\mathrm{e}^{-D \phi\left(x_{0}, t_{0}\right) \cdot y}-1-\left(D \phi\left(x_{0}, t_{0}\right) \cdot y\right) \mathbb{I}_{\{|y|<1\}}\right\} J(y) \mathrm{d} y \\
& +\varepsilon+o_{\delta}(1) .
\end{aligned}
$$

It only remains to pass to the limit as $\varepsilon, \delta \rightarrow 0$. Since $J$ satisfies (2.1) and (2.4), the integral over $\mathbb{R}^{N}$ converges, and we can send $M$ to $+\infty$ and obtain in the limit:

$$
\begin{aligned}
\frac{\partial \phi}{\partial t}\left(x_{0}, t_{0}\right) \leqslant & -\operatorname{Tr}\left(A \cdot D \phi\left(x_{0}, t_{0}\right) \otimes D \phi\left(x_{0}, t_{0}\right)\right)-B \cdot D \phi\left(x_{0}, t_{0}\right) \\
& -\int_{\mathbb{R}^{N}}\left\{\mathrm{e}^{-D \phi\left(x_{0}, t_{0}\right) \cdot y}-1-\left(D \phi\left(x_{0}, t_{0}\right) \cdot y\right) \mathbf{I}_{\{|y|<1\}}\right\} J(y) \mathrm{d} y,
\end{aligned}
$$

which shows that $\bar{I}^{A}$ at $\left(x_{0}, t_{0}\right)$ is a subsolution in the sense of viscosity.

Case 2. The point $\left(x_{0}, t_{0}\right)$ is located at the boundary, $x_{0} \in \partial B_{1}, t_{0}>0$. Then the sequence $\left(x_{n}, t_{n}\right)$ may have points $x_{n}$ either inside $B_{1} \times(0, \infty)$, or at the boundary, or even outside $B_{1}$. If $x_{n} \in B_{1}$, we use the equation as in the previous case while if $x_{n} \in \partial B_{1}$, we use the relaxed boundary condition in Definition 2.2. Finally, if $\left|x_{n}\right|>1$, then $I_{R_{n}}\left(x_{n}, t_{n}\right)=0$ so that in any case, one has

$$
\min \left\{\partial_{t} \phi+H(D \phi) ; I_{R_{n}}^{A}\right\} \leqslant 0 \text { at }\left(x_{n}, t_{n}\right) .
$$


We then pass to the limit as $n \rightarrow+\infty$ and get the relaxed condition for $\bar{I}^{A}$ at the boundary.

Case 3. The point $\left(x_{0}, t_{0}\right)$ is located at $t_{0}=0, x_{0} \in B_{1}$. The same happens as in Case 2: we have either $t_{n}=0$ and then we use the initial condition, or $t_{n}>0$ in which case we use the equation. In any case we get

$$
\min \left\{\partial_{t} \phi+H(D \phi) ; I_{R_{n}}^{A}-A\right\} \leqslant 0 \text { at }\left(x_{n}, t_{n}\right),
$$

which gives in the limit

$$
\min \left\{\partial_{t} \phi+H(D \phi) ; \bar{I}^{A}-A\right\} \leqslant 0 \text { at }\left(x_{0}, t_{0}\right) .
$$

Now, it is well known (see for instance [3, Thm. 4.7]) that in this case, the initial condition is equivalent to $\lim _{t \rightarrow 0} \bar{I}^{A} \leqslant A$. Actually this can be proved as in Proposition 6.5, using that in the present situation, the Hamiltonian is finite everywhere.

Conclusion: First, the supersolution conditions for $\underline{I}^{A}$ are obtained by the same method, with reversed inequalities. Then using comparison between u.s.c./l.s.c. sub/super solutions for (2.9), we get the inequality $\underline{I}^{A} \leqslant \bar{I}^{A}$, which implies equality of both functions. Hence, the sequence converge uniformly in $\bar{B}_{1} \times[0, T]$ for all $T>0$ to the unique solution $I^{A}$.

3.3. Proof of Theorem 3.1] Conclusion. This result only comes from the fact that for any $A>0$, by construction,

$$
\bar{I}^{A}=\inf (\bar{I}, A), \quad \underline{I}^{A}=\inf (\underline{I}, A),
$$

with

$$
\bar{I}(x, t):=\limsup _{R \rightarrow \infty}{ }^{*} I_{R}(x, t), \quad \underline{I}(x, t):=\liminf _{R \rightarrow \infty} * I_{R}(x, t) .
$$

The fact that $\bar{I}^{A}=\underline{I}^{A}$, together with Lemma 2.13, yields the result for fixed $(x, t)$, passing to the limit as $A \rightarrow \infty$.

Now, the convergence of $I_{R}$ to $I_{\infty}$ is locally uniform in $\bar{B}_{1} \times[0, \infty)$ so that for any $T>0$ as long as $x / R \leqslant 1$ and $0 \leqslant t / R \leqslant T$,

$$
\left|I_{R}(x / R, t / R)-I_{\infty}(x / R, t / R)\right|=o(1) \rightarrow 0 \quad \text { as } \quad R \rightarrow \infty,
$$

where $o(1)$ is uniform with respect to $x$ and $t$ as above. Thus estimate (3.1) indeed holds, which ends the proof. Notice that at $t=0$, both $I_{R}$ and $I_{\infty}$ are infinite (which corresponds to $A=+\infty$ ), but anyway, the difference $I_{R}-I_{\infty}$ remains uniformly controlled.

Remark 3.4. At this stage we have an estimate valid up to the boundary of $B_{R}$. In the next sections we shall derive more explicit estimates only for $|x| \leqslant \theta R$, with $\theta \in(0,1)$, because we use the asymptotic behaviour of $L(q)$ as $|q| \rightarrow \infty$.

\section{Compactly Supported Kernels}

In this section we prove that a general " $R \ln R$ " bound is valid for compactly supported kernels, extending the symmetric and regular case proved in [12. In order to take into account the possible asymmetry of the kernel, we define below for any unit vector $\nu$, the size of the support of $J$ in the direction $\nu$ :

Definition 4.1. For any $\nu \in \mathbb{R}^{N}$ with $|\nu|=1$, let

$$
\rho(\nu):=\sup \{r>0: J(r \nu)>0\} .
$$


Notice that since $J$ is continuous, for any $r>0$ close enough to $\rho(\nu), J$ is positive in a neighborhood of $r \nu$. Notice also that if $J$ is symmetric, then $\rho(\nu)=\rho$, the radius of the support of $J$. We shall first derive a bound from below for nonsymmetric kernels in the logarithmic scale:

Lemma 4.2. Let $J$ be a continuous compactly supported kernel, let $\nu=p /|p|$ and define $\rho(\nu)$ as above. Then we have:

$$
\liminf _{|p| \rightarrow \infty} \frac{\ln \left(p \cdot D H^{\text {ess }}(p)\right)}{\rho(\nu)|p|} \geqslant 1 .
$$

Proof. Let us first choose a unit vector $\nu$, define $\rho(\nu)$ by (4.1) and consider $p \in \mathbb{R}^{N}$ going to infinity in this direction: $p /|p|=\nu$ and $|p| \rightarrow \infty$. We begin by writing

$$
p \cdot D H^{\mathrm{ess}}(p)=\int_{\{p \cdot y \leqslant 0\} \cap\left\{|y|>\rho_{0} / 2\right\}} p \cdot y \mathrm{e}^{p \cdot y} J(y) \mathrm{d} y+\int_{\{p \cdot y>0\} \cap\left\{|y|>\rho_{0} / 2\right\}} p \cdot y \mathrm{e}^{p \cdot y} J(y) \mathrm{d} y .
$$

The first term is bounded from below by $-c|p|$ and for the second one we define for $\varepsilon>0$ and $\beta<1$, the set

$$
\mathcal{C}_{\varepsilon, \beta}^{+}=\left\{y: \frac{\rho(\nu)}{\beta} \leqslant|y| \leqslant \rho(\nu), p \cdot y \geqslant(1-\varepsilon)|p||y| \geqslant 0\right\} \cap\left\{|y|>\rho_{0} / 2\right\} .
$$

Hence

$$
\begin{aligned}
\int_{\{p \cdot y>0\} \cap\left\{|y|>\rho_{0} / 2\right\}} p \cdot y \mathrm{e}^{p \cdot y} J(y) \mathrm{d} y & \geqslant|p| \frac{\rho(\nu)}{\beta}(1-\varepsilon) \mathrm{e}^{(1-\varepsilon)|p| \frac{\rho(\nu)}{\beta}} \int_{\mathcal{C}_{\varepsilon, \beta}^{+}} J(y) \mathrm{d} y \\
& \geqslant C(\varepsilon, \beta)|p| \frac{\rho(\nu)}{\beta}(1-\varepsilon) \mathrm{e}^{(1-\varepsilon)|p| \frac{\rho(\nu)}{\beta}} .
\end{aligned}
$$

Notice that $C(\varepsilon, \beta)>0$ since $J$ is continuous and positive near $\rho(\nu) \nu$, even if this constant could be small. Summing up,

$$
\begin{aligned}
p \cdot D H^{\mathrm{ess}}(p) & \geqslant C(\varepsilon, \beta)|p| \frac{\rho(\nu)}{\beta}(1-\varepsilon) \mathrm{e}^{(1-\varepsilon)|p| \frac{\rho(\nu)}{\beta}}-|p| c \\
& \geqslant K C(\varepsilon, \beta)|p| \frac{\rho(\nu)}{\beta}(1-\varepsilon) \mathrm{e}^{(1-\varepsilon)|p| \frac{\rho(\nu)}{\beta}}
\end{aligned}
$$

for some constant $K$. Therefore, we obtain for every $\beta$ and $\varepsilon$,

$$
\begin{aligned}
\liminf _{|p| \rightarrow \infty} \frac{\ln \left(p \cdot D H^{\mathrm{ess}}(p)\right)}{\rho(\nu)|p|} & \geqslant \liminf _{|p| \rightarrow \infty}\left(\frac{\ln C(\varepsilon, \beta)}{\rho(\nu)|p|}+\frac{\ln \left(\frac{\rho(\nu)}{\beta}|p|(1-\varepsilon)\right)}{\rho(\nu)|p|}+\frac{\frac{\rho(\nu)}{\beta}|p|(1-\varepsilon)}{\rho(\nu)|p|}\right) \\
& =\frac{1-\varepsilon}{\beta} .
\end{aligned}
$$

Now, letting $\varepsilon \rightarrow 0$ and $\beta \rightarrow 1$ we conclude that (4.2) holds.

In other words, using Lemmas 2.6 and 2.8, we have obtained

$$
\liminf _{|q| \rightarrow \infty} \frac{\ln L(q)}{\rho(\nu)\left|p_{0}(q)\right|} \geqslant 1 \text {. }
$$

Then in order to have a more explicit bound using Theorem 3.1 , we shall compare with a symmetric kernel, then using the radius of the support of $J$.

Theorem 4.3. Let $J$ be a compactly supported kernel satisfying (2.1). We denote by $\rho$ the size of the support of $J$ :

$$
\rho=\inf \left\{r>0: \operatorname{supp}(J) \subset B_{r}\right\} .
$$


Then the following estimate holds: for any $\theta \in(0,1)$ and $T>0$, as $R \rightarrow \infty$,

$$
\sup _{\substack{|x| \leqslant \theta R \\ 0 \leqslant t \leqslant T R}}\left|u-u_{R}\right|(x, t) \leqslant \mathrm{e}^{-\frac{(1-\theta)}{\rho} R \ln R+o(R \ln R)} .
$$

Notice that $J$ can be asymmetric and have a singularity at the origin.

Proof. We first use Lemma 2.10 to reduce our estimate to the case of symmetric, compactly supported kernels. More precisely, using Lemma 2.10, if $J_{*}$ is a symmetric kernel such that $J \prec J_{*}$ and $\operatorname{supp}\left(J_{*}\right)=B_{\rho}$, then for $|p|$ big enough, $L_{*} \leqslant L$ where $L_{*}$ is the Lagrangian associated to $J_{*}$. Taking a look at Theorem 3.1, this implies that

$$
\begin{aligned}
-R I_{\infty}(x / R, t / R) & =-R \min _{y \in \partial B_{1}}(t / R) L\left(\frac{x / R-y}{t / R}\right) \\
& \leqslant-R \min _{y \in \partial B_{1}}(t / R) L_{*}\left(\frac{x / R-y}{t / R}\right) \\
& \leqslant-\min _{y \in \partial B_{R}} t L_{*}\left(\frac{x-y}{t}\right) .
\end{aligned}
$$

Now we assume that $|x|<\theta R$ so that in this set $|x-y| \geqslant(1-\theta) R \rightarrow \infty$ and we shall use the behaviour at infinity of $L_{*}$. Lemma 2.8 allows us to wipe out the possible singular part near the origin as well as the differential terms of the Hamiltonian.

Since $J_{*}$ is symmetric, so is $L_{*}^{\text {ess }}$ so that, noting $L_{*}^{\text {ess }}(x)=\underline{L}_{*}(|x|)$ we get

$$
\liminf _{R \rightarrow \infty} \frac{-R I_{\infty}(x / R, t / R)}{-\min _{y \in \partial B_{R}} t \underline{L}_{*}\left(\frac{|x-y|}{t}\right)} \leqslant 1 .
$$

Then we use the results of [12, Lemma 4.1 and Corollary 4.2] applied to $\underline{L}_{*}$ which is symmetric, associated to a nonsingular kernel to conclude the proof.

\section{INTERMEDIATE KERNELS}

We now consider a general kernel $J$ satisfying (2.1), positive everywhere in $\mathbb{R}^{N}$, so that we can always write

$$
J(y)=\mathrm{e}^{-|y| \omega(y)}, \text { with } \omega(y)=-\frac{\ln J(y)}{|y|} .
$$

We will now make some further assumptions in this section:

$$
\left\{\begin{array}{l}
J \text { is } \mathrm{C}^{1} \text {-smooth for }|y|>0, \\
|D J(y)| \text { is bounded on }\{|y|>\varepsilon\}, \forall \varepsilon>0, \\
y \mapsto|y| \omega(y) \text { is superlinear and convex, } \\
\exists \eta \in(0,1], \liminf _{|y| \rightarrow+\infty} \frac{(y \cdot D \omega(y))}{|y||D \omega(y)|} \geqslant \eta .
\end{array}\right.
$$

Let us comment on these hypotheses:

(i) The regularity assumption on $J$ (which implies the same regularity for $\omega$ ) is not crucial since by comparison we can deal with less regular kernels, using the results of Section 2 . 
(ii) About the convexity of $|y| \omega(y)$, it is actually only required for large $|y|$ for the same reason: we only care about the tail of $J$.

(iii) The case of compactly supported kernels, which would correspond to $\omega(y)=+\infty$ outside a ball, is treated in Section 4 . On the other hand, the case of critical kernels treated in Section 6 corresponds to $\lim \omega(y)=$ $\ell<\infty$. So, the assumption of superlinearity is in between: $\lim \omega(y)=\infty$. This is why we speak of intermediate kernels here.

(iv) The superlinearity assumption implies that $J$ automatically satisfies (2.4) since indeed, for any $\beta>0$, we have that $\omega(y)>\beta$ for $|y|$ large enough.

$(v)$ The convexity and superlinearity assumptions altogether allow us to define the Legendre transform $\mathcal{K}(\cdot)$ of $|y| \omega(y)$, which will also be superlinear and convex; see [36]:

$$
\mathcal{K}(p):=\sup _{y \in \mathbb{R}^{N}}\{p \cdot y-|y| \omega(y)\} .
$$

This function $\mathcal{K}(\cdot)$ will play a big role in estimating the rate of convergence. Notice that it is also the Legendre transform of $\ln (1 / J)$.

(vi) The "angle" condition on $D \omega$ says that the gradient cannot take a purely tangential position. This is a very weak assumption in this form that allows us to derive a minimum behaviour for nonsymmetric kernels.

5.1. Properties of $\mathcal{K}$. Thanks to Lemma 2.6, we know that

$$
L(q) \sim p_{0}(q) \cdot D H\left(p_{0}(q)\right),
$$

where $p_{0}(q)$ is such that $L(q)=p_{0} \cdot q-H\left(p_{0}\right)$. Thus, a main step consists in finding a lower bound for $p \cdot D H(p)$. Here is where $\mathcal{K}(p)$ plays an important role: roughly speaking, we will see that

$$
\text { " } p \cdot D H(p)=\int_{\mathbb{R}^{N}} p \cdot y \mathrm{e}^{p \cdot y-|y| \omega(y)} \mathrm{d} y \geqslant \mathrm{e}^{\mathcal{K}(p) " .} .
$$

Hence a detailed study of the properties of $\mathcal{K}$ is needed. To this aim, let $y_{0}(p)$ be the point where the sup in (5.2) is attained.

Lemma 5.1. Let us assume that $J(y)=\mathrm{e}^{-|y| \omega(y)}$ satisfies (2.1) and (5.1). Then the function $\mathcal{K}$ is nonnegative and $\mathcal{K}(0)=0$. Moreover, for $|p|$ large enough, the supremum in (5.2) is attained at a unique point $y_{0}=y_{0}(p)$ and $\left|y_{0}(p)\right| \rightarrow \infty$ as $|p| \rightarrow \infty$.

Proof. For $p \in \mathbb{R}^{N}$ fixed, let us denote by

$$
\varphi_{p}(y):=p \cdot y-|y| \omega(y) .
$$

Then observe that $\varphi(0)=0$, so that $\mathcal{K}(p)=\sup \varphi_{p} \geqslant 0$ and of course $\mathcal{K}(0)=0$. The superlinearity $y \mapsto|y| \omega(y)$ implies that $\varphi_{p}(y) \rightarrow-\infty$ as $|y| \rightarrow \infty$, so that there exists at least a maximum point $y_{0}(p)$. Now, any maximum point satisfies:

$$
p=D(|y| \omega(y))\left(y_{0}\right)=\frac{D J\left(y_{0}\right)}{J\left(y_{0}\right)},
$$

and by our assumptions, $J(y) \rightarrow 0$ as $|y| \rightarrow \infty$ while $|D J|$ remains bounded away from $y=0$. Thus, as $|p| \rightarrow \infty$, necessarily $\left|y_{0}(p)\right| \rightarrow+\infty$.

We shall now prove that for $|p|$ large enough, the maximum point $y_{0}(p)$ is unique. Since $y \mapsto|y| \omega(y)$ is convex for large $|y|$, say $|y|>C$, then $\varphi_{p}$ is concave for $|y|>C$, independently of $p$. Indeed, this comes from the fact that $D^{2} \varphi_{p}(y)=-D^{2}(|y| \omega(y))$. 
Thus we take $M>0$ large enough so that for any $|p|>M$, any minimum point $y_{0}(p)$ satisfies $\left|y_{0}(p)\right|>C$ and enters the region where $\varphi_{p}$ is concave. Then, the only case when there may exist several maximum points is the case when $\varphi_{p}$ would be constant on some open set. But since $\omega(y) \rightarrow+\infty$ as $|y| \rightarrow \infty$, this cannot happen for $y$ large. Hence if $|p|$ is large, the maximum is attained at a unique point $y_{0}(p)$.

Lemma 5.2. Under hypothesis (5.1), we have

$$
\liminf _{|p| \rightarrow+\infty} \frac{\left(p \cdot y_{0}(p)\right)}{|p|\left|y_{0}(p)\right|} \geqslant \eta \text {. }
$$

Proof. By the definition of $y_{0}(p)$, we have $p=D(|y| \omega(y))\left(y_{0}\right)$ so that

$$
|p| \leqslant \omega\left(y_{0}\right)+\left|y_{0}\right|\left|D \omega\left(y_{0}\right)\right|
$$

and

$$
\left(y_{0} \cdot p\right)=\left|y_{0}\right| \omega\left(y_{0}\right)+\left|y_{0}\right|\left(y_{0} \cdot D \omega\left(y_{0}\right)\right) .
$$

This implies, using (5.1), that

$$
\left(y_{0} \cdot p\right) \geqslant \eta\left(\left|y_{0}\right| \omega\left(y_{0}\right)+\left|y_{0}\right|^{2}\left|D \omega\left(y_{0}\right)\right|\right)=\eta\left|y_{0}\right||p| ;
$$

hence the result follows by taking the liminf.

5.2. Estimating $L(q)$. We begin by a technical lemma that will help us in constructing a box where $p \cdot y-|y| \omega(y)$ is close to $\mathcal{K}(p)$ (see below):

Lemma 5.3. There exists $M>0$ such that for any $|p|>M$ and any $\xi \in$ $B\left(y_{0}(p), 1 /|p|\right)$, we have

$$
\frac{(D \omega(\xi) \cdot \xi)}{|D \omega(\xi)||\xi|} \geqslant \frac{3 \eta}{4}>0
$$

with $\eta$ defined in (5.1).

Proof. Notice first that since $\xi$ is at distance at most $1 /|p|$ of $y_{0}$ (which goes to infinity as $|p| \rightarrow \infty)$, then all the points $\xi \in B\left(y_{0}(p), 1 /|p|\right)$ verify that $|\xi|$ is big provided $|p|$ is also big. More precisely, using (5.1) we know that there exists an $m>0$ such that for any $|y|>m$,

$$
\frac{(D \omega(y) \cdot y)}{|D \omega(y)||y|} \geqslant \frac{3 \eta}{4}>0 .
$$

Now, since $\left|y_{0}(p)\right| \rightarrow \infty$ as $|p| \rightarrow \infty$, there exists $M>0$ such that if $|p|>M$, then any $|\xi| \in B\left(y_{0}(p), 1 /|p|\right)$ satisfies $|\xi|>\left|y_{0}\right|-1 /|p|>m$. Then we may apply the above estimate by taking $y=\xi$, which gives the result.

Now let us make some definitions clear.

Definition 5.4. The angle between two vectors $a, b \in \mathbb{R}^{N}$ is defined as follows:

$$
\operatorname{ang}(a, b)=\arccos \frac{(a \cdot b)}{|a||b|} \in[0, \pi] .
$$

Moreover, given a vector $a \in \mathbb{R}^{N}$ and an angle $\alpha \in[0, \pi / 2]$, we define the positive cone $\mathcal{C}^{+}$in the direction $a$ with aperture $\alpha$ as follows:

$$
\mathcal{C}^{+}(a, \alpha)=\left\{b \in \mathbb{R}^{N}: \operatorname{ang}(a, b) \leqslant \alpha\right\} .
$$

Accordingly we define the negative cone as follows:

$$
\mathcal{C}^{-}(a, \alpha)=\mathcal{C}^{+}(-a, \alpha) .
$$


Notice that by considering only apertures $0 \leqslant \alpha \leqslant \pi / 2$ (which is enough for our purpose here), we make sure that $\mathcal{C}^{-}(a, \alpha) \cap \mathcal{C}^{+}(a, \alpha)=\{0\}$. Then we have the following lemma:

Lemma 5.5. Let us consider the cone $\mathcal{C}^{*}(p):=\mathcal{C}^{-}\left(y_{0}(p), \pi / 2-\arccos (\eta / 2)\right)$ and

$$
\mathcal{A}(p)=\left\{y_{0}(p)+z:|z| \leqslant \frac{1}{|p|}, z \in \mathcal{C}^{*}(p)\right\} .
$$

Then for $|p|$ big enough, we have

$$
\forall y \in \mathcal{A}(p), \quad p \cdot y-|y| \omega(y) \geqslant p \cdot y_{0}-\left|y_{0}\right| \omega\left(y_{0}\right)-1 .
$$

Moreover, the volume of $\mathcal{A}(p)$ is bounded by

$$
\mathcal{A}(p) \mid \geqslant \frac{c(N, \eta)}{|p|^{N}}
$$

for some constant $c(N, \eta)>0$.

Proof. In all of the proof we consider at least $|p|>M$ so that we may apply Lemma 5.3 and take $y \in \mathcal{A}(p)$. Then $y \in B\left(y_{0}, \frac{1}{|p|}\right)$ so that we have $(p \cdot y) \geqslant\left(p \cdot y_{0}\right)-1$.

Moreover, since $0<\eta \leqslant 1$, the aperture of the cone $\mathcal{C}^{*}(p), \arccos (\eta / 2)$, is strictly positive and not equal to $\pi / 2$. Hence for $|p|$ big enough, $\mathcal{A}(p) \subset B_{\left|y_{0}\right|}$ so that $|y| \leqslant\left|y_{0}\right|$ and it is enough to check that $\omega(y) \leqslant \omega\left(y_{0}\right)$ to get what we want, that is:

$$
(p \cdot y)-|y| \omega(y) \geqslant\left(p \cdot y_{0}\right)-\left|y_{0}\right| \omega\left(y_{0}\right)-1
$$

To this end, we write

$$
\omega(y)-\omega\left(y_{0}\right)=D \omega(\xi) \cdot\left(y-y_{0}\right)
$$

for some $\xi \in\left[y, y_{0}\right]$. The point is that, unless we are in a symmetric case, we do not have a very precise estimate for $D \omega(\xi)$. But we will prove that if $y \in \mathcal{C}^{*}(p)$, then necessarily

$$
D \omega(\xi) \cdot\left(y-y_{0}\right) \leqslant 0
$$

which will be enough to get the desired estimate.

We shall first show that the image set $D \omega\left(B_{1 /|p|}\left(y_{0}(p)\right)\right)$ is contained in a cone of aperture comparable to $\arccos (3 \eta / 4)$, in the direction $y_{0}$. More precisely, we claim that for any $\xi \in B\left(y_{0}, 1 /|p|\right)$,

$$
0 \leqslant \operatorname{ang}\left(D \omega(\xi), y_{0}\right) \leqslant \arccos (3 \eta / 4)+o\left(1 /|p|^{1 / 2}\right) ;
$$

see Figure 1. Indeed, we first estimate the angle $\operatorname{ang}\left(y_{0}, \xi\right)$ for any $\xi=y_{0}+z \in$ $B\left(y_{0}, 1 /|p|\right)$ as follows:

$$
\begin{aligned}
\frac{\left(y_{0} \cdot \xi\right)}{\left|y_{0}\right||\xi|} & =\frac{\left(y_{0} \cdot y_{0}\right)}{\left|y_{0}\right|\left(\left|y_{0}\right|+O(1 /|p|)\right)}+\frac{\left(y_{0} \cdot z\right)}{\left|y_{0}\right|\left(\left|y_{0}\right|+O(1 /|p|)\right)} \\
& =1-o(1 /|p|)
\end{aligned}
$$

(recall that $\left.\left|y_{0}(p)\right| \rightarrow \infty\right)$, where the term $o(1 /|p|)$ is nonnegative. The expansion, as $x \rightarrow 0^{-}, \arccos (1+x)=O\left(x^{1 / 2}\right)$ implies that

$$
\operatorname{ang}\left(y_{0}, \xi\right)=o\left(1 /|p|^{1 / 2}\right)
$$

and finally, we use the fact that

$$
\operatorname{ang}(D \omega(\xi), y) \leqslant \operatorname{ang}(D \omega(\xi), \xi)+\operatorname{ang}\left(\xi, y_{0}\right) \leqslant \arccos (3 \eta / 4)+o\left(1 /|p|^{1 / 2}\right) .
$$




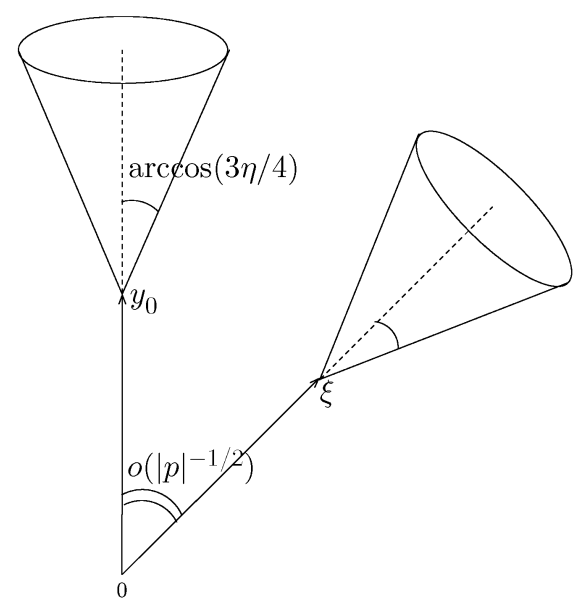

Figure 1. Cones in $y_{0}$ and $\xi$.

Thus, for $|p|$ large enough (recall that the arccos function is strictly decreasing on $(0,1))$,

$$
\operatorname{ang}\left(D \omega(\xi), y_{0}\right) \leqslant \arccos (\eta / 2) .
$$

Figure 2 shows the vectorial cone of aperture $\arccos (3 \eta / 4)$ in the direction $\xi$ which is included in the vectorial cone of aperture $\arccos (\eta / 2)$ in the direction $y_{0}$. Hence, if we choose a point $y=y_{0}+z$ such that $z \in \mathcal{C}^{-}\left(y_{0}, \pi / 2-\arccos (\eta / 2)\right)$ we make sure that $\operatorname{ang}(D \omega(\xi), z) \geqslant \pi / 2$, which yields

$$
D \omega(\xi) \cdot z=D \omega(\xi) \cdot\left(y-y_{0}\right) \leqslant 0 .
$$

To end the lemma, we only need to mention that $\mathcal{A}(p)$ is given by the intersection of the ball $B\left(y_{0}, 1 /|p|\right)$ with a cone placed at $y_{0}$, of aperture $\pi / 2-\arccos (\eta / 2)$. Hence its volume is indeed given by $c(N, \eta) /|p|^{N}$ for some constant $c(N, \eta)>0$.

Remark 5.6. As $\eta$ becomes close to zero, the aperture of the cone $\mathcal{C}^{*}(p)$ becomes very small: $\pi / 2-\arccos (\eta / 2) \sim 0$. So in the limit case $\eta=0$ we would not be able to construct a nonempty set $\mathcal{A}(p)$, at least with this method. Now, the size of $\mathcal{A}(p)$ gets small as $|p| \rightarrow \infty$ since it behaves like $|p|^{-N}$, but nevertheless this will be sufficient to get a suitable estimate, since inside this (small) region, we will get an exponential behaviour.

Lemma 5.7. Let $J(y)=\mathrm{e}^{-|y| w(y)}$ satisfying (2.1) and (5.1). Then the following estimate holds:

$$
\liminf _{|p| \rightarrow \infty} \frac{\ln \left(p \cdot D H^{\mathrm{ess}}(p)\right)}{\mathcal{K}(p)} \geqslant 1
$$

Proof. We start from

$$
\begin{aligned}
p \cdot D H^{\mathrm{ess}}(p) & =\int_{\left\{|y|>\rho_{0} / 2\right\}} p \cdot y \mathrm{e}^{p \cdot y-|y| w(y)} \mathrm{d} y \\
& =\int_{\{p \cdot y \leqslant 0\} \cap\left\{|y|>\rho_{0} / 2\right\}}(\ldots) \mathrm{d} y+\int_{\{p \cdot y>0\} \cap\left\{|y|>\rho_{0} / 2\right\}}(\ldots) \mathrm{d} y,
\end{aligned}
$$




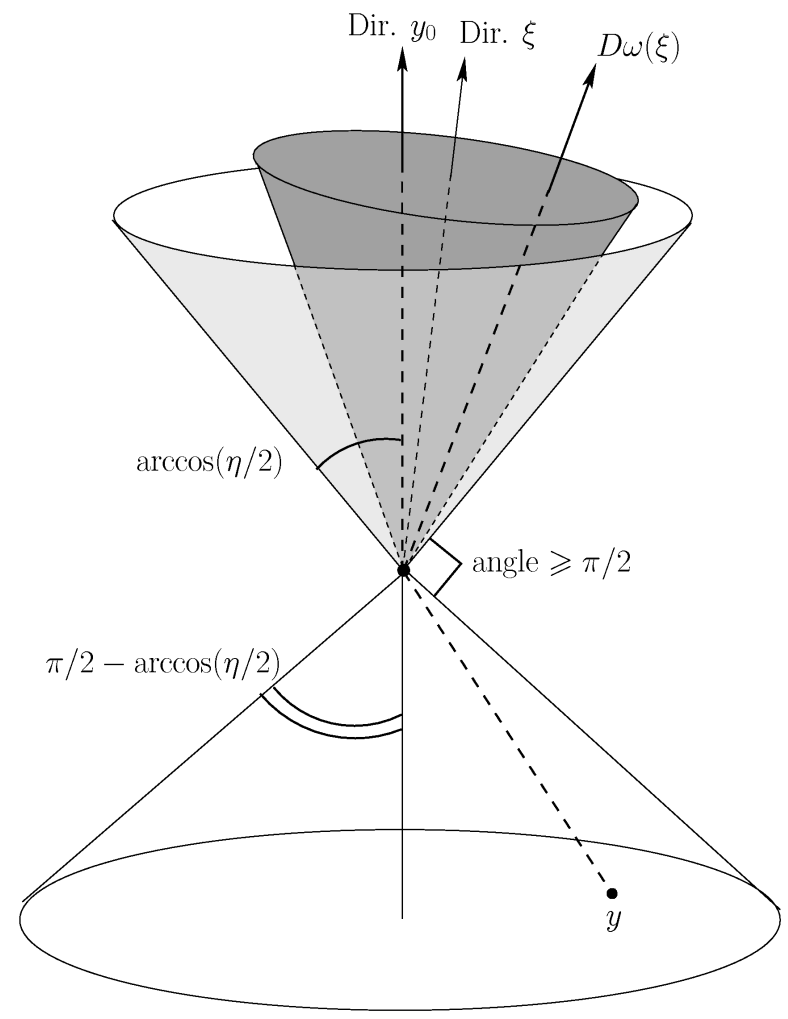

FIguRE 2. Position of the vectorial cones.

and the integral over $\{p \cdot y \leqslant 0\} \cap\left\{|y|>\rho_{0} / 2\right\}$ is bounded by $C|p|$ for some $C>0$, so that it will be negligible (recall that $p \cdot D H^{\mathrm{ess}}(p)$ behaves at least exponentially; see Lemma 2.5). Thus we shall give an estimate of $p \cdot D H^{\text {ess }}(p)$ only in terms of the integral over $\{p \cdot y>0\} \cap\left\{|y|>\rho_{0} / 2\right\}$ : for $|p|$ large enough, we have

$$
p \cdot D H^{\mathrm{ess}}(p) \geqslant \frac{1}{2} \int_{\{p \cdot y>0\} \cap\left\{|y|>\rho_{0} / 2\right\}} p \cdot y \mathrm{e}^{p \cdot y-|y| \omega(y)} \mathrm{d} y .
$$

Now let us notice that, for $|p|$ big enough, $\mathcal{A}(p) \subset\{p \cdot y>0\} \cap\left\{|y|>\rho_{0} / 2\right\}$. So we can write, using Lemma 5.5 and (5.1):

$$
\begin{aligned}
p \cdot D H^{\mathrm{ess}}(p) & \geqslant \frac{1}{2} \int_{\mathcal{A}(p)} p \cdot y \mathrm{e}^{p \cdot y-|y| \omega(y)} \mathrm{d} y \\
& \geqslant \frac{1}{2} \eta|p| \int_{\mathcal{A}(p)}|y| \mathrm{e}^{\mathcal{K}(p)-1} \mathrm{~d} y \\
& \geqslant \frac{1}{2} \eta|p||\mathcal{A}(p)|\left(\left|y_{0}(p)\right|-\frac{1}{|p|}\right) \mathrm{e}^{\mathcal{K}(p)-1} .
\end{aligned}
$$

Hence, taking logarithms and dividing by $\mathcal{K}(p)$ :

$$
\frac{\ln \left(p \cdot D H^{\text {ess }}(p)\right)}{\mathcal{K}(p)} \geqslant \frac{\ln (\eta|p| / 2)}{\mathcal{K}(p)}+\frac{\ln |\mathcal{A}(p)|}{\mathcal{K}(p)}+\frac{\ln \left(\left|y_{0}(p)\right|-1 /|p|\right)}{\mathcal{K}(p)}+1-\frac{1}{\mathcal{K}(p)} .
$$


Recall that $\mathcal{K}(p)$ is superlinear, $\left|y_{0}(p)\right| \rightarrow \infty$ and $|\mathcal{A}(p)| \geqslant c|p|^{-N}$ to conclude that

$$
\liminf _{|p| \rightarrow \infty} \frac{\ln \left(p \cdot D H^{\mathrm{ess}}(p)\right)}{\mathcal{K}(p)} \geqslant 1
$$

We assume now that $J_{*}(y)=\mathrm{e}^{-|y| \omega_{*}(y)}$ is symmetric in order to get a more precise estimate. We denote by $L_{*}$ and $\mathcal{K}_{*}$ the associated Lagrangian and Legendre transform of $|y| \omega_{*}(y)$. Since in this case $\mathcal{K}_{*}$ is symmetric (and still superlinear), we know that for $|p|$ large enough, $\mathcal{K}_{*}^{-1}: \mathbb{R}_{+} \rightarrow \mathbb{R}^{N}$ is defined and for any $p \in \mathcal{K}_{*}^{-1}(|z|)$, we have $|p|=$ constant.

Proposition 5.8. Let us assume that $J_{*}$ is a symmetric kernel satisfying (2.1) and (5.1). Then

$$
\liminf _{|q| \rightarrow \infty} \frac{L_{*}(q)}{|q|\left|\mathcal{K}_{*}^{-1}(\ln |q|)\right|} \geqslant 1 .
$$

Proof. In this proof, we drop the $*$-subscript for simplicity. We use estimate (5.5) and, since $J$ is symmetric, $H^{\text {ess }}(\cdot)$ is also symmetric. Hence $p_{0} \cdot D H^{\text {ess }}\left(p_{0}\right)=$ $\left|p_{0}\right|\left|D H^{\text {ess }}\left(p_{0}\right)\right|$ so that, using that $\mathcal{K}(\cdot)$ is superlinear:

$$
\liminf _{|q| \rightarrow \infty} \frac{\ln \left|p_{0}\right|+\ln \left|D H^{\text {ess }}\left(p_{0}\right)\right|}{\mathcal{K}\left(p_{0}\right)}=\liminf _{|q| \rightarrow \infty} \frac{\ln \left|D H^{\text {ess }}\left(p_{0}\right)\right|}{\mathcal{K}\left(p_{0}\right)} \geqslant 1 .
$$

In other words, this means that for any $\varepsilon \in(0,1)$, if $|q|$ is large enough,

$$
\ln |q| \geqslant(1-\varepsilon) \mathcal{K}\left(p_{0}(q)\right) \text {. }
$$

Using again that $\mathcal{K}$ is superlinear and symmetric, we have that for any $\varepsilon \in(0,1)$, it is enough to take $|q|$ big to get

$$
\left|p_{0}(q)\right| \leqslant\left|\mathcal{K}^{-1}\left((1-\varepsilon)^{-1} \ln |q|\right)\right| .
$$

From this we get that for any $\varepsilon \in(0,1)$ :

$$
\liminf _{|q| \rightarrow \infty} \frac{p_{0} \cdot D H^{\mathrm{ess}}\left(p_{0}\right)}{\left|q \| \mathcal{K}^{-1}\left((1-\varepsilon)^{-1} \ln |q|\right)\right|} \geqslant 1
$$

and we pass to the limit as $\varepsilon \rightarrow 0$ to get the result for $L^{\text {ess }}(q) \sim p_{0} \cdot D H^{\text {ess }}\left(p_{0}\right)$ :

$$
\liminf _{|q| \rightarrow \infty} \frac{L^{\text {ess }}(q)}{|q|\left|\mathcal{K}^{-1}(\ln |q|)\right|} \geqslant 1 .
$$

Finally, we invoke Lemma 2.8 to conclude that the result holds for $L(q)$.

5.3. Conclusion. We are now ready to prove one of the main results of this paper:

Theorem 5.9. Let $J(y)=\mathrm{e}^{-|y| w(y)}$ be a kernel satisfying (2.1) and (5.1). Let us consider a symmetric kernel $J_{*}(y)=\mathrm{e}^{-|y| \omega_{*}(y)}$ such that $J \prec J_{*}$, and denote by $\mathcal{K}_{*}$ the associated Legendre transform of $|y| \omega_{*}(y)$. Then the following estimate holds as $R \rightarrow \infty$ : for any $\theta \in(0,1), T>0$,

$$
\sup _{\substack{|x| \leqslant \theta R \\ 0 \leqslant t \leqslant T R}}\left|u-u_{R}\right|(x, t) \leqslant \mathrm{e}^{-(1-\theta) R \mathcal{K}_{*}^{-1}(\ln ((1-\theta) R / t))(1+o(1))} .
$$


Proof. The proof essentially follows the one in the case of compactly supported kernels: we first use Lemma 2.10 to reduce our estimate to the case of a symmetric kernel $J \prec J_{*}$ :

$$
\begin{aligned}
-R I_{\infty}(x / R, t / R) & =-R \min _{y \in \partial B_{1}}(t / R) L\left(\frac{x / R-y}{t / R}\right) \\
& \leqslant-R \min _{y \in \partial B_{1}}(t / R) L_{*}\left(\frac{x / R-y}{t / R}\right) \\
& \leqslant-t \underline{L}_{*}\left(\frac{\operatorname{dist}\left(x ; \partial B_{R}\right)}{t}\right)
\end{aligned}
$$

where $\underline{L}_{*}(|x|)=L_{*}(x)$ is the symmetric Lagrangian associated to $J_{*}$.

Now we assume that $|x|<\theta R$ so that in this set $\operatorname{dist}\left(x ; \partial B_{R}\right) \geqslant(1-\theta) R \rightarrow \infty$ and we use the behaviour at infinity of $L_{*}$ given by (5.6) to get the result.

Corollary 5.10. In particular, if $x$ remains in a bounded set $B_{M}$, we can take any $\theta>0$ and if $t$ also remains bounded we obtain a simpler estimate:

$$
\sup _{|x|<M, 0<t<1}\left|u-u_{R}\right|(x, t) \leqslant \mathrm{e}^{-R \mathcal{K}_{*}^{-1}(\ln R)(1+o(1))} .
$$

Several remarks are to be made:

(i) The authors gave some explicit estimates in [12, which consist here more or less in making explicit the function $\mathcal{K}(\cdot)$. We refer to Section 7.1 for a list of known explicit behaviours.

(ii) Even if we are able to prove a lower estimate for asymmetric kernels Lemma (5.7) - using $\mathcal{K}(p)$ which is not symmetric in general, we are facing a problem: knowing the behaviour of $p \cdot D H(p)$ is not enough to know the behaviour of each of the vectors, unless we make sure that they point more or less in the same direction, and this is not clear unless the kernel is "almost" symmetric because of the exponential behaviour. This is why we compare with the smallest symmetric kernel above $J$ in order to have a more explicit behaviour.

(iii) Even if we were able to derive a bound taking into account the asymmetry, then we would have to study the min in Theorem 3.1, which is again not obvious unless we have an almost symmetric Lagrangian.

(iv) However, see Section 7.2 for the 1-D case where we can deal with asymmetric kernels, since the regions $\{x>0\}$ and $\{x<0\}$ are clearly separated.

\section{CRitical kernels}

We now assume that $J$ is symmetric and that

$$
J(y)=\mathrm{e}^{-|y| \omega(y)}, \text { where } \lim _{|y| \rightarrow \infty} \omega(y)=\ell .
$$

Hence $J$ satisfies (2.5) with $\beta_{0}=\ell$. We want to show that the estimate remains valid even if $H$ is not finite everywhere. To this aim, we have to study the HamiltonJacobi equation more carefully. In this case, the domain of definition of the Hamiltonian $H$ is exactly: $\operatorname{dom}(H)=B_{\ell}$, that is, $H=+\infty$ outside $B_{\ell}$. For simplicity, we will first assume that $\ell=\beta_{0}=1$, the adaptations for other values being straightforward. Then we shall give the general result in Theorem 6.9. 
6.1. Hamilton-Jacobi equation with nonfinite Hamiltonian. We study the equation $u_{t}+H(D u)=0$, posed in the cylinder $Q:=B_{1} \times(0, \infty)$ (although most of the results of this section would also hold for more general cylinders). Here, we assume that the Hamiltonian $H$ is infinite in the complement of $B_{1}$, which is the main difficulty. Following [4, we begin by constructing a new equation which is equivalent in the viscosity sense to $u_{t}+H(D u)=0$, the main interest being that it allows us to prove a comparison result and analyze the initial trace of the solutions.

On the parabolic boundary

$$
\partial_{P} Q=\left(\partial B_{1} \times[0, \infty)\right) \cup\left(B_{1} \times\{t=0\}\right),
$$

we impose a continuous boundary condition $f$ in the viscous sense. More precisely, we consider the following problem:

$$
\begin{cases}u_{t}+H(D u)=0 & \text { in } B_{1} \times(0, \infty), \\ u(x, t)=f(x, t) & \text { on } \partial B_{1} \times[0, \infty), \\ u(x, 0)=f(x, 0) & \text { in } B_{1}\end{cases}
$$

Definition 6.1. Given $f \in \mathrm{C}\left(\partial_{P} Q\right)$, we say that an upper semi-continuous function $u$ is a viscosity subsolution of (6.1) if for any smooth function $\varphi$ such that $u-\varphi$ reaches a maximum at $\left(x_{0}, t_{0}\right)$ we have:

$$
\begin{aligned}
\left(x_{0}, t_{0}\right) \in Q & \Rightarrow \varphi_{t}+H(D \varphi) \leqslant 0 \quad \text { at }\left(x_{0}, t_{0}\right), \\
\left(x_{0}, t_{0}\right) \in \partial_{P} Q & \Rightarrow \min \left(\varphi_{t}+H(D \varphi) ; u-f\right) \leqslant 0 \quad \text { at }\left(x_{0}, t_{0}\right) .
\end{aligned}
$$

The same definition holds with reversed inequalities (and min instead of max) for an upper semi-continuous viscosity sub-solution. Finally:

Definition 6.2. A locally bounded function $u: Q \rightarrow \mathbb{R}$ is a viscosity solution of (6.1) if its upper semi-continuous envelope is a super-solution and its lower semicontinuous envelope is a sub-solution of (6.1).

Let us mention that in the case when $H$ is finite everywhere, solutions take on the initial data in a classical way. But since here some data may not be compatible with the fact that $\operatorname{dom}(H)=B_{1}$, this implies that a boundary/initial layer appears, and this is precisely the phenomenon we are facing. In order to understand this layer, we first need to reinterpret the equation with a new Hamiltonian:

Proposition 6.3. Sub-solutions and super-solutions of (6.1) are also sub-solutions and super-solutions (in the viscous sense) of the equation:

$$
\max \left(u_{t}+H(D u) ;|D u|-1\right)=0
$$

with the same data on the parabolic boundary.

Proof. Let $u$ be a viscosity sub-solution and consider a smooth test function $\varphi$ such that $u-\varphi$ has a maximum at $\left(x_{0}, t_{0}\right)$. We assume for simplicity that $\left(x_{0}, t_{0}\right) \in Q$ (the argument being similar if it is a boundary point). Then since by definition

$$
\partial_{t} \varphi\left(x_{0}, t_{0}\right)+H(D \varphi)\left(x_{0}, t_{0}\right) \leqslant 0,
$$

we have necessarily $H(D \varphi)<+\infty$, so that $|D \varphi| \leqslant 1$ and thus $u$ also satisfies (in the viscous sense) the inequation

$$
\max \left(\partial_{t} u+H(D u) ;|D u|-1\right) \leqslant 0 .
$$


Now if $v$ is a super-solution and $\varphi$ is such that $v-\varphi$ has a minimum at $\left(x_{0}, t_{0}\right)$, then

implies that

$$
\partial_{t} \varphi\left(x_{0}, t_{0}\right)+H(D \varphi)\left(x_{0}, t_{0}\right) \geqslant 0
$$

$$
\max \left(\partial_{t} \varphi\left(x_{0}, t_{0}\right)+H(D \varphi)\left(x_{0}, t_{0}\right) ;\left|D \varphi\left(x_{0}, t_{0}\right)\right|-1\right) \geqslant 0,
$$

which implies that $v$ is a super-solution of (6.2).

Proposition 6.4. Let $u$ be a viscosity sub-solution and $v$ be a viscosity supersolution of

$$
\max \left(u_{t}+H(D u) ;|D u|-1\right)=0
$$

with $u \leqslant v$ on the parabolic boundary $\partial_{P} Q$. Then $u \leqslant v$.

Proof. Formally speaking, if one fixes $\mu \in(0,1)$ and considers a maximum point $\left(x_{0}, t_{0}\right)$ of $\mu u-v$, then if $|D v|\left(x_{0}, t_{0}\right) \geqslant 1$, since by definition $\mu D u=D v$ at $\left(x_{0}, t_{0}\right)$, it happens that $|D u|\left(x_{0}, t_{0}\right) \geqslant 1 / \mu>1$, which is impossible since $u$ is a viscosity subsolution. So we have both $|D v|,|D u|<1$ at $\left(x_{0}, t_{0}\right)$ and we do the comparison as always, using standard viscosity techniques. Now let us be more precise.

We fix $\mu \in(0,1)$, and $T>0$ and consider a point $\left(x_{0}, y_{0}, t_{0}, s_{0}\right) \in{\overline{B_{1}}}^{2} \times[0, T]^{2}$, where

$$
\Phi:(x, y, t, s) \mapsto \mu u(x, t)-v(y, s)-\frac{|x-y|^{2}}{\varepsilon^{2}}-\frac{|t-s|^{2}}{\beta^{2}}-\frac{C}{T-t}
$$

reaches its maximum. We assume that it is an interior point; otherwise, using the boundary values one immediately obtains $\Phi \leqslant 0$ in $B_{1}^{2} \times(0, T)^{2}$, which is what we want.

Fixing one variable, since $(x, t) \mapsto \Phi\left(x, y_{0}, t, s_{0}\right)$ reaches its maximum at $\left(x_{0}, t_{0}\right)$ one may consider the following test function for $u$ at $\left(x_{0}, t_{0}\right)$ :

$$
\phi_{1}(x, t):=\frac{1}{\mu}\left(v\left(y_{0}, s_{0}\right)+\frac{\left|x-y_{0}\right|^{2}}{\varepsilon^{2}}+\frac{\left|t-s_{0}\right|^{2}}{\beta^{2}}+\frac{C}{T-t}\right) .
$$

Indeed, if we denote by $p:=2\left(x_{0}-y_{0}\right) / \varepsilon^{2}, q:=2\left(t_{0}-s_{0}\right) / \beta^{2}$, it happens that

$$
\max \left(\frac{\mu^{-1} C}{(T-t)^{2}}+\mu^{-1} q+H\left(\mu^{-1} p\right) ;\left|\mu^{-1} p\right|-1\right) \leqslant 0 .
$$

On the other hand, for $v$ we use at $\left(y_{0}, s_{0}\right)$ the test function

$$
\phi_{2}(s, t):=\mu u\left(x_{0}, t_{0}\right)-\frac{\left|x_{0}-y\right|^{2}}{\varepsilon^{2}}-\frac{\left|t_{0}-s\right|^{2}}{\beta^{2}},
$$

which leads to

$$
\max (q+H(p) ;|p|-1) \geqslant 0 .
$$

If we assume that $|p| \geqslant 1$, then $\mu^{-1}|p| \geqslant \mu^{-1}>1$, which is impossible from (6.3). So, both $|p|$ and $\mu^{-1}|p|$ are less than 1 and then the proof follows standard arguments of viscosity solutions: we can combine (6.3) and (6.4), getting rid of the max which gives (after multiplying the first inequality by $\mu$ ):

$$
\frac{C}{(T-t)^{2}}+\mu H\left(\mu^{-1} p\right)-H(p) \leqslant 0 .
$$


We claim that $h(\mu):=\mu H\left(\mu^{-1} p\right)-H(p) \geqslant 0$ for any $p \in \mathbb{R}^{N}$ and $\mu \in(0,1)$, which leads to a contradiction with (6.5), so that an interior maximum of $\Phi$ is impossible. Hence, $\Phi \leqslant 0$ in $\left(B_{1}\right)^{2} \times(0, T)^{2}$ and since $\beta, \varepsilon, C, T, \mu$ are arbitrary, we finally conclude that $u \leqslant v$ in $B_{1} \times(0, \infty)$.

To end the proof, let us check the claim: using the convexity of $H$, one gets

$$
h^{\prime}(\mu)=-\frac{1}{\mu}\left(\left(\mu^{-1} p\right) \cdot D H\left(\mu^{-1} p\right)-H\left(\mu^{-1} p\right)\right) \leqslant 0,
$$

and since $h(1)=0$, we see that $h \geqslant 0$ for $\mu \in(0,1)$.

Proposition 6.5. Let $u$ be a viscosity solution of $\max \left(u_{t}+H(D u) ;|D u|-1\right)=0$, and $u=f$ on $\partial_{P} Q$. Then the initial trace of $u, u(0)$ satisfies:

$$
\max (u(0)-f ;|D u(0)|-1)=0 .
$$

Proof. Let $u$ be a subsolution of the equation with boundary data $f$. Then at $t=0$,

$$
\min \left(\max \left(u_{t}+H(D u),|D u|-1\right) ; u-f\right) \leqslant 0 .
$$

We take a test function $\varphi(x, t)=C_{\varepsilon} t+\left|x-x_{0}\right|^{2} / \varepsilon^{2}$ such that

$$
\max \left(\varphi_{t}+H(D \varphi),|D \varphi|-1\right) \geqslant 0 .
$$

This is always possible if $\varepsilon$ is small enough, so that this implies $u(0) \leqslant f$. Thus:

$$
\max (u(0)-f ;|D u(0)|-1) \leqslant 0 .
$$

Now, we consider a super-solution $u$ and take $\varphi(x)$ such that $f-\varphi$ has a minimum at $x_{0}$. Then for any $C>0$,

$$
\psi:(x, t) \mapsto u(x, 0)-\varphi(x)+C t
$$

has a maximum at $t=0, x=x_{0}$. Using $\psi$ as a test function, we obtain

$$
\max (\max (-C+H(D \varphi(0)),|D \varphi(0)|-1) ; u-f) \geqslant 0 .
$$

For $C$ big enough, we have $-C+H(D \varphi(0))<0$ so that there remain two possibilities:

Case 1. $|D \varphi(0)-1|<0$, which implies $u-f \geqslant 0$ so that:

$$
\max (\varphi(0)-f ;|D \varphi(0)|-1) \geqslant 0 .
$$

Case 2. $|D \varphi(0)-1| \geqslant 0$, which implies

$$
\max (\varphi(0)-f ;|D \varphi(0)|-1) \geqslant 0 .
$$

So if $u$ is a solution, both inequalities give the equality.

Remark 6.6. Equation (6.6) can be understood as an obstacle problem: both $|D u(0)| \leqslant 1, u(0) \leqslant f$ and $(u(0)-f)(|D u(0)|-1)=0$. 
6.2. Back to the estimate of $I$. As in Section 3 we define for any $A>0$ :

$$
I_{R}^{A}:=-\frac{1}{R} \ln \left(\mathrm{e}^{-R A}+I\right), \quad \bar{I}^{A}:=\limsup _{R \rightarrow \infty} I_{R}^{A}, \quad \underline{I}^{A}:=\liminf _{R \rightarrow \infty} I_{R}^{A} .
$$

Taking liminf and limsup and a test function, we see that for any $A>0, \bar{I}^{A}$ and $\underline{I}^{A}$ are respectively sub- and super-solutions of

$$
\max \left(u_{t}+H(D u) ;|D u|-1\right)=0, \quad I^{A}(0)=A .
$$

Consequently, using (6.4), we obtain that $\bar{I}^{A}=\underline{I}^{A}$, so that as $R \rightarrow+\infty$, every sequence $I_{R}^{A}$ converges to the unique solution $I^{A}$ of the problem. Then as $A \rightarrow+\infty$, $I^{A} \rightarrow I$, which satisfies the equation with $I(0, x)=\operatorname{dist}\left(x ; \partial B_{1}\right)$.

Now we have to identify the limit $I$, and to do so we have to study some properties of this specific Lagrangian.

Lemma 6.7. The Lagrangian L satisfies the following properties:

$$
|D L(q)|<1 \text { and } L(q) \sim|q| \text { as }|q| \rightarrow \infty .
$$

Proof. Since by definition,

$$
L(q)=\sup _{p \in \mathbb{R}^{N}}\{p \cdot q-H(p)\}=\sup _{|p|<1}\{p \cdot q-H(p)\},
$$

and $H(p) \rightarrow+\infty$ as $|p| \rightarrow 1$, this implies that the sup is attained at some $\left|p_{0}(q)\right|<1$. On the other hand, a simple calculus shows that $D L(q)=p_{0}(q)$, so that indeed, for any $q,|D L(q)|<1$. This also implies a first basic estimate: $L(q) \leqslant|q|$. To get the equivalent result, we first bound $H(p)$. Let us first notice that

$$
H(p)=H^{\mathrm{ess}}(p)+f(p)=H^{\mathrm{ess}}(p)+O(1)
$$

indeed the singular and differential parts of the Hamiltonian remain bounded in the set $\{|p|<1\}$, as well as $D f(p)$. Now, for any $|p|<1$,

$$
\begin{aligned}
H(p) \leqslant f(p)+\int_{\mathbb{R}^{N}} \mathrm{e}^{(|p|-1)|y|} \mathrm{d} y & \leqslant f(p)+c(N) \int_{0}^{\infty} \mathrm{e}^{(|p|-1) r} r^{N-1} \mathrm{~d} r \\
& =f(p)+\frac{c(N)(N-1) !}{(1-|p|)^{N}} .
\end{aligned}
$$

Hence,

$$
L(q) \geqslant \sup _{|p|<1}\left\{p \cdot q-\frac{c(N)(N-1) !}{(1-|p|)^{N}}-f(p)\right\},
$$

and this sup is attained for $p_{0}=p_{0}(q)$ satisfying the equation:

$$
q=|p| c(N)(N-1) !(1-|p|)^{-N-1} \frac{p}{|p|}+D f(p) .
$$

Thus, as $|q| \rightarrow \infty$, necessarily $\left|p_{0}\right| \rightarrow 1$ and since $p_{0}$ and $q$ point in the same direction,

$$
L(q) \geqslant \sup _{|p|<1}\left\{p \cdot q-\frac{c(N)(N-1) !}{(1-|p|)^{N}}-f(p)\right\} \sim p_{0}(q) \cdot q \sim|q| .
$$

Since we have seen that $L(q) \leqslant|q|$, we conclude that

$$
L(q) \sim|q| \text { as }|q| \rightarrow \infty .
$$


Since $J$ is assumed to be symmetric, so are $H$ and $L$ and we write $\underline{L}(|x|)=L(x)$.

Proposition 6.8. The solution of (6.1) with initial data $I(0, x)=\operatorname{dist}\left(x ; \partial B_{1}\right)$ and $I=0$ on the boundary is given by the Lax-Oleinik formula:

$$
I(x, t)=t \underline{L}\left(\frac{\operatorname{dist}\left(x ; \partial B_{1}\right)}{t}\right) .
$$

Proof. Since $|D L|<1$, then $|D I|<1$ so that the compatibility condition is always fulfilled and the equation holds everywhere. Now we take a look at the initial data. Since $L(q) \sim|q|$, this implies:

$$
t \underline{L}\left(\frac{\operatorname{dist}\left(x ; \partial B_{1}\right)}{t}\right) \rightarrow \operatorname{dist}\left(x ; \partial B_{1}\right) \text { as } t \rightarrow 0,
$$

so that indeed the Lax-Oleinik formula gives a solution. Since the viscosity solution is unique, this ends the proof.

The reader will easily check that if $\ell=\beta_{0} \neq 1$, then all the results of this section remain valid and then $L(q) \sim \beta_{0}|q|$ as $|q| \rightarrow \infty$. Moreover, Lemma 2.8 and Lemma 2.10 are also valid in the present case since $|p|$ remains bounded: as we have seen, $H(p)=H^{\text {ess }}(p)+O(1)$ as $|p|=\beta_{0}$.

Hence we may write a more general result for possibly nonsymmetric and singular kernels:

Theorem 6.9. Let $J$ be a kernel satisfying (2.1) and (2.5). In particular, $J$ can be asymmetric and have a singularity at the origin. Then for any $\theta \in(0,1)$, we have the following estimate as $R \rightarrow \infty$ : for any $\theta \in(0,1), T>0$,

$$
\sup _{\substack{|x| \leqslant \theta R \\ 0 \leqslant t \leqslant T R}}\left|u-u_{R}\right|(x, t) \leqslant \mathrm{e}^{-(1-\theta) \beta_{0} R+o(R)} .
$$

Proof. We skip the details since this is the same as for Proposition 4.3. we first reduce the estimate to a symmetric kernel by comparison, putting a symmetric kernel above $J$ with the same $\beta_{0}$ in (2.5) and then we wipe out the possible singularities and differential terms. Actually, the proof is even simpler since those terms remain bounded in the set $\left\{|p|<\beta_{0}\right\}$; hence only the exponential part of the Hamiltonian plays a role in the estimate.

Remark 6.10. As $\beta_{0} \rightarrow 0$, the estimate gets worse. Indeed, this means that the kernel tends to behave slower than an exponential and we are facing a problem of fat tails (like a power decay), which this method cannot handle.

\section{Further Results AND COMMEnts}

7.1. Explicit bounds. In some particular cases, we already gave concrete estimates in [12, by directly studying the Hamiltonian $H$. Computing the function $\mathcal{K}^{-1}$, here we recover these estimates under the following form:

$$
\sup _{|x|<M, 0<t<1}\left|u-u_{R}\right|(x, t) \leqslant \mathrm{e}^{-R \mathcal{K}^{-1}(\ln R)(1+o(1))}
$$

Table 1 collects some known asymptotic behaviours of $\mathcal{K}^{-1}$ and $\sup \left(u-u_{R}\right)$. Notice that since $\mathcal{K}$ is superlinear (except in the critical case), then $\mathcal{K}^{-1}(z)$ is defined for $z>0$ big enough. Most of the calculations are straightforward; we only sketch the case $J(y)=\mathrm{e}^{\mathrm{e}^{-y}}$ : in this case, $\mathcal{K}(y) \sim|y| \ln |y|$ and if we define $z=|y| \ln |y|$ we get $\ln z=\ln |y|+\ln \ln |y| \sim \ln |y|$. Hence $z \sim|y| \ln z$, which implies 
TABLE 1. Behaviour of $\mathcal{K}^{-1}$

\begin{tabular}{ccc}
\hline Kernel & $\mathcal{K}^{-1}(z)$ & $\sup \left|u-u_{R}\right|$ \\
\hline$J(y)=\mathbb{I}_{\{|y| \leqslant \rho\}}(y)$ & $z \rho^{-1}$ & $\mathrm{e}^{-R \ln (R) / \rho}$ \\
$J(y)=\mathrm{e}^{-\mathrm{e}^{|y|}}$ & $z(\ln z)^{-1}$ & $\mathrm{e}^{-R(R \ln R)}$ \\
$J(y)=\mathrm{e}^{-|y|^{\alpha}}, \alpha>1$ & $z^{(\alpha-1) / \alpha}$ & $\mathrm{e}^{-R(\ln R)^{(\alpha-1) / \alpha}}$ \\
$J(y)=\mathrm{e}^{-|y| \ln |y|}$ & $\ln z$ & $\mathrm{e}^{-R \ln \ln R}$ \\
$J(y)=\mathrm{e}^{-\alpha|y|}, \alpha>0$ & $\alpha$ & $\mathrm{e}^{-\alpha R}$
\end{tabular}

$|y| \sim z / \ln z$. Let us also mention that in the case $J(y)=\mathrm{e}^{-\alpha|y|}, \mathcal{K}^{-1}(z)=\alpha$ in the sense of graphs.

Remark 7.1. As we have seen, the presence of a singularity at the origin does not modify the behaviour of $\mathcal{K}^{-1}$, so for instance, if we multiply by $|y|^{-2}$ any of the previous kernels we obtain the same estimate.

7.2. Nonsymmetric kernels in one space dimension. As we have seen, it is in general difficult to give an explicit behaviour of $\sup \left|u-u_{R}\right|$ for nonsymmetric kernels, unless we compare with a symmetric one. But in the case $N=1$, the regions where $\{x>0\}$ and $\{x<0\}$ are clearly separated so that more precise estimates can be given according to the tails of $J$ at $-\infty$ and $+\infty$ which can be different. We shall just illustrate this in an explicit case which concerns the most extreme cases we can cover: on the one side we have a compactly supported kernel, while on the other side, we have an exponential decay.

Proposition 7.2. Let us assume that $N=1$ and $J$ is given by:

$$
J(y):=\frac{1}{2} \mathrm{e}^{-|y|} \mathbf{I}_{\{y<0\}}+\frac{1}{2} \mathbb{I}_{\{y \geqslant 0\}} .
$$

Then the associated Lagrangian satisfies:

$$
L(q) \underset{q \rightarrow+\infty}{\sim} q \ln q \text { and } L(q) \underset{q \rightarrow-\infty}{\sim} q .
$$

Proof. A straightforward calculus shows that the Hamiltonian is defined in the region $\{p>-1\}$ and that:

$$
H(p)=\frac{\mathrm{e}^{p}}{2 p}-\frac{1}{2 p}+\frac{1}{2(p+1)}-1 .
$$

For $q>0$, we calculate the Lagragian as follows:

$$
L(q)=\sup _{p>-1}\{p q-H(p)\}=\sup _{p>0}\{p q-H(p)\} .
$$

Indeed, if $q>0$, then $p q>0$ only for $p>0$ and we know that the sup is nonnegative so that it has to be attained for $p>0$. With this remark, the estimate is just the same as for the case when $J(y)=\frac{1}{2} \mathbb{I}_{\{y \geqslant 0\}}$. The same remark holds when $q<0$ : the sup is attained in the region $-1<p<0$ and the behaviour is given by the exponential decay of $J$. 
Then we are able to use Theorem 3.1 in a more precise way (we consider $x$ fixed in a bounded domain for simplicity):

Proposition 7.3. Let $J$ be defined as above. Then

$$
\begin{aligned}
\sup _{0<x<M, 0<t<1}\left|u-u_{R}\right|(x, t) & \leqslant \mathrm{e}^{-R \ln R(1+o(1))}, \\
\sup _{-M<x<0,0<t<1}\left|u-u_{R}\right|(x, t) & \leqslant \mathrm{e}^{-R(1+o(1))}
\end{aligned}
$$

Proof. We just come back to the expression of $I_{\infty}$ :

$$
I_{\infty}(x / R, t / R)=\min _{y \in \partial B_{R}} \frac{t}{R} L\left(\frac{R x-y}{t}\right) .
$$

So, if $1<x<M$, whether $y=-R$ or $y=+R$, we always have $R x-y \geqslant$ $(M-1) R \rightarrow+\infty$. Hence the min of $L$ is attained for $y=-R$ and we recover the behaviour of $L(q)$ for $q=R(M+1) \rightarrow \infty$, that is, an $R \ln R$ behaviour. On the other hand, if $-M<x<-1$, the min is attained for $y=-R$ and we get the linear behaviour of $L(q)$ for $q \rightarrow-\infty$.

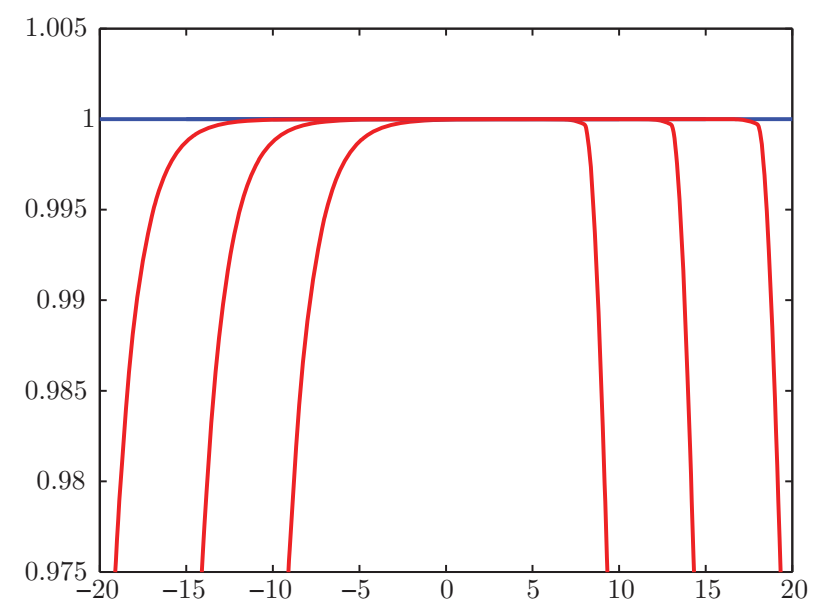

FiguRE 3. Convergence for nonsymmetric kernel.

In Figure 3 we plot for $u_{0}=1$ the approximations $u_{R}$ for $R=10,15,20$ and nonsymmetric $J$ as in Proposition 7.2. This illustrates the different rate of convergence whether $x<0$ or $x>0$.

7.3. KPP-type results. In this section, we briefly explain how our results allow us to treat a nonlocal version of the KPP-problem (Kolmogorov-Petrovskii-Piskounov) associated to equation (1.1) with the classical monostable $u(1-u)$-term. For simplicity we shall just explain this on the following equation:

$$
\partial_{t} u-(J * u-u)=u(1-u) \quad \text { in } \quad \mathbb{R}^{N} \times[0, T]
$$

with a continuous initial data $u_{0}(x)=g(x), 0 \leqslant g \leqslant 1$. Existence of solutions with initial data $0 \leqslant g \leqslant 1$ may be obtained for instance by Perron's method. 
In order to study convergence of $u$ to the equilibrium states 0 and 1 for large $x$ and $t$, the following scaling is widely used:

$$
u^{\varepsilon}(x, t)=u\left(\frac{x}{\varepsilon}, \frac{t}{\varepsilon}\right) .
$$

It turns out here that, formally, $u^{\varepsilon}$ satisfies the equation

$$
\partial_{t} u^{\varepsilon}-\frac{1}{\varepsilon}\left(J_{\varepsilon} * u^{\varepsilon}-u^{\varepsilon}\right)=\frac{u^{\varepsilon}\left(1-u^{\varepsilon}\right)}{\varepsilon} \text { in } \mathbb{R}^{N} \times[0, T],
$$

with $J_{\varepsilon}(x)=\varepsilon^{N} J(x / \varepsilon)$, so that one may use exactly the same method as was used in Section 3 with $\varepsilon$ playing the role of $1 / R \rightarrow 0$.

We may thus combine the techniques of [5] with the ones we used in Section 3 to handle the convergence of the nonlocal term, which give us some estimates at which convergence to the states $u=0$ and $u=1$ occur. In fact, once we know how to deal with the nonlocal terms of the equation, the rest of the proof only follows [5] this is why we only sketch a proof below. Here we denote by $H$ the following Hamiltonian:

$$
H(p):=\int_{\mathbb{R}^{N}}\left(\mathrm{e}^{p \cdot y}-1\right) J(y) \mathrm{d} y,
$$

where it is assumed that $J \in \mathrm{C}\left(\mathbb{R}^{N}\right)$ and satisfies (2.5) for some $\beta_{0}>0$.

Theorem 7.4. Let $G_{0}=\{g(x)=0\}$ and $G_{1}=\{g(x)=1\}$. Then the following results hold: (i) Let $I_{0}^{\varepsilon}=-\varepsilon \ln \left(u^{\varepsilon}\right)$. Then $I_{0}^{\varepsilon} \rightarrow I_{0}$, the solution of the variational inequality:

$$
\min \left(\frac{\partial I_{0}}{\partial t}+H\left(D I_{0}\right)+1, I_{0}\right)=0 \quad \text { in } \quad \mathbb{R}^{N} \times(0, \infty)
$$

and

$$
I_{0}(x, 0)= \begin{cases}+\infty & \text { if } x \in G_{0}, \\ 0 & \text { if } x \in \mathbb{R}^{N} \backslash G_{0} .\end{cases}
$$

(ii) Let $I_{1}^{\varepsilon}:=-\varepsilon \ln \left(1-u^{\varepsilon}\right)$. Then $I_{1}^{\varepsilon} \rightarrow I_{1}$ locally uniformly in the set $\mathcal{C}=$ $\left\{I_{0}(x, t)=0\right\}$, where

$$
\left\{\begin{array}{lll}
\frac{\partial I_{1}}{\partial t}+H\left(D I_{1}\right)+1=0 & \text { in } & \operatorname{int} \mathcal{C}, \\
I_{1}(x, t)=0 & \text { on } \quad & \partial \mathcal{C} \cap\{t>0\}, \\
I_{1}(x, 0)=+\infty \mathbb{I}_{\left\{G_{1}\right\}}(x) . & &
\end{array}\right.
$$

Sketch of proof. For (i), it turns out that $u^{\varepsilon}$ satisfies the equation

$$
\partial_{t} u^{\varepsilon}-\frac{1}{\varepsilon}\left(J_{\varepsilon} * u^{\varepsilon}-u^{\varepsilon}\right)=\frac{u^{\varepsilon}\left(1-u^{\varepsilon}\right)}{\varepsilon} \quad \text { in } \mathbb{R}^{N} \times[0, T],
$$

with initial data $u^{\varepsilon}(x, 0)=g(x / \varepsilon)$, where the rescaled kernel is $J_{\varepsilon}(x)=\varepsilon^{N} J(x / \varepsilon)$.

Then we make the log-transform

$$
I_{0}^{\varepsilon, A}(x, t)=-\varepsilon \ln \left(u^{\varepsilon}(x, t)+\exp \left(-\frac{A}{\varepsilon}\right)\right),
$$

which satisfies

$$
\partial_{t} I_{0}^{\varepsilon, A}+\int\left(\mathrm{e}^{-\frac{1}{\varepsilon}\left\{I_{0}^{\varepsilon, A}(x+y \varepsilon)-I_{0}^{\varepsilon, A}(x)\right\}}-1\right) J(y) \mathrm{d} y=-\frac{u^{\varepsilon}\left(1-u^{\varepsilon}\right)}{u^{\varepsilon}+\exp (-A / \varepsilon)} .
$$


Passage to the limit on the left-hand side is done exactly as in Section 3 while handling the right-hand side follows exactly from KPP classical techniques: first notice that by construction, $I_{0}^{\varepsilon, A} \geqslant 0$. Then, if in the limit $I_{0}^{A}(x, t)=\lim _{\varepsilon \rightarrow 0} I_{0}^{\varepsilon, A}>$ 0 , this means that $u^{\varepsilon} \rightarrow 0$ so that clearly as $\varepsilon \rightarrow 0$, the right-hand side converges to 1.

For (ii), we set similarly

$$
I_{1}^{\varepsilon, A}(x, t)=-\varepsilon \ln \left(1-u^{\varepsilon}(x, t)+\exp \left(-\frac{A}{\varepsilon}\right)\right) .
$$

The first step consists in proving that $\overline{I_{1}^{A}}:=\limsup _{\varepsilon \rightarrow 0} I_{1}^{\varepsilon, A}$ and $I_{1}^{A}:=$ $\liminf _{\varepsilon \rightarrow 0} I_{1}^{\varepsilon, A}$ are respectively sub- and super-solutions of the variational inequality:

$$
\begin{aligned}
\max \left(\partial_{t} I+H(D I)+1, I-\psi^{A}\right) & =0 \text { in } \mathbb{R}^{N} \times(0, \infty), \\
I(x, 0) & =A \cdot \mathbb{I}_{\left\{G_{1}\right\}}(x),
\end{aligned}
$$

where $\psi^{A}(x, t)=1$ if $(x, t) \in \mathcal{C}$ and 0 otherwise. Direct comparison between $\overline{I_{1}^{A}}$ and $I_{1}^{A}$ cannot be derived here since no information on the regularity of $\mathcal{C}$ is available. The final result then follows from a representation formula for $I_{1}^{A}$. We refer to [5] for the details.

Then, coming back to the original variables, one can obtain explicit exponential convergence rates, which follows from our study of the asymptotic behaviour of the Lagrangian associated to $H$ (as was done in Sections 4 , 5, 6).

7.4. Relation with optimal existence results. We would like to add another final comment on a related subject. As was said, by estimating $\sup _{B_{R}}\left|u-u_{R}\right|(x, T)$, we are measuring the total number of processes that can escape the box $B_{R}$ between $t=0$ and $t=T$. Another way of understanding this is that we are somehow estimating the Green kernel associated to the equation.

Thus, in [13, the authors together with R. Ferreira are deriving similar estimates but in the context of optimal initial data, which is also a way of measuring the behaviour of the kernel at infinity. Hence it is not so surprising that similar estimates appear, even if they are obtained through a totally different method.

For instance, it turns out that if $J$ is compactly supported, the optimal class of existence for $u_{t}=J * u-u$ in $\mathbb{R}^{N}$ consists of initial data satisfying:

$$
\left|u_{0}(x)\right| \leqslant C \mathrm{e}^{|x| \ln |x|}
$$

hence we recover an $R \ln R$ estimate for the Green function, typical of compactly supported kernels. We refer to 13 , for more results in this direction.

\section{ACKNowledgments}

Both authors were partially supported by project MTM2008-06326-C02-02 (Spain). We would like to thank Guy Barles for his help and advice during this work, and the referee for pointing out some interesting remarks. 


\section{REFERENCES}

[1] O. Alvarez, A. Tourin, Viscosity solutions of nonlinear integro-differential equations. Ann. Inst. H. Poincaré Anal. Non Linéaire 13 (1996), no. 3, 293-317. MR1395674 (97d:35023)

[2] D. Applebaum, Lévy processes and stochastic calculus. Second edition. Cambridge Studies in Advanced Mathematics, 116. Cambridge University Press, Cambridge, 2009. MR 2512800 (2010m:60002)

[3] G. BARles, Solutions de viscosité des équations de Hamilton-Jacobi. Mathématiques \&5 Applications 17, Springer-Verlag, Paris, 1994. MR1613876 (2000b:49054)

[4] G. Barles, An approach of deterministic control problems with unbounded data. Ann. Inst. H. Poincaré Anal. Non Linéaire 7 (1990), no. 4, 235-258. MR1067774(91h:49035)

[5] G. Barles, P. Souganidis, A remark on the asymptotic behavior of the solution of the KPP equation. C. R. Acad. Sci. Paris Sér. I Math. 319 (1994), no. 7, 679-684. MR1300069 (95k:35105)

[6] G. Barles, Ch. Daher, M. Romano, Convergence of numerical schemes for parabolic equations arising in finance theory, Math. Models Methods Appl. Sci. 5 (1995), no. 1, 125143. MR1315000 (95m:90031)

[7] G. Barles, E. Chasseigne, C. Imbert, Dirichlet boundary conditions for second order elliptic non-linear integro-differential equations, Indiana Univ. Math. J. 57 (2008), no. 1, 213-246. MR2400256 (2009b:35088)

[8] G. Barles, C. Imbert, Second-order elliptic integro-differential equations: viscosity solutions' theory revisited. Ann. Inst. H. Poincaré Anal. Non Linéaire 25 (2008), no. 3, 567-585. MR:2422079 (2009c:35102)

[9] G. Barles, S. Mirrahimi, B. Perthame, Concentration in Lotka-Volterra parabolic or integral equations: a general convergence result, Methods Appl. Anal. 16 (2009), no. 3, 321-340. MR 2650800 (2011g:35017)

[10] G. Barles, L.C. Evans, P.E. Souganidis, Wavefront propagation for reaction-diffusion systems of PDE, Duke Math. J. 61 (1990), no. 3, 835-858. MR1084462 (91k:35119)

[11] J. Bertoin, Lévy processes. Cambridge Tracts in Mathematics 121, Cambridge University Press, Cambridge, 1996. MR.1406564 (98e:60117)

[12] C. Brändle, E. Chasseigne, Large deviations estimates for some non-local equations. Fast decaying kernels and explicit bounds. Nonlinear Analysis 71 (2009), 5572-5586. MR2560225 (2010k:60099)

[13] C. Brändle, E. Chasseigne, R. Ferreira, Unbounded solutions of the nonlocal heat equation, Commun. Pure Appl. Anal. 10 (2011), no. 6, 1663-1686. MR.2805332 (2012e:35257)

[14] X. Cabré, J.-M. Roquejoffre, Front propagation in Fisher-KPP equations with fractional diffusion, C. R. Math. Acad. Sci. Paris 347 (2009), no. 23-24, 1361-1366. MR 2588782 (2010j:35577)

[15] L. Caffarelli, L. Silvestre, An extension problem related to the fractional Laplacian. Comm. Partial Differential Equations 32 (2007), no. 7-9, 1245-1260. MR2354493 (2009k:35096)

[16] L. Caffarelli, L. Silvestre, Regularity theory for fully nonlinear integro-differential equations. Comm. Pure Appl. Math. 62 (2009), no. 5, 597-638. MR2494809 (2010d:35376)

[17] P. Carr, H. Geman, D. Madan, M. Yor, The Fine Structure of Asset Returns: An Empirical Investigation, Journal of Business 75 (2002), 2, 305-332.

[18] E. Chasseigne, M. Chaves, J.D. Rossi, Asymptotic behavior for nonlocal diffusion equations. J. Math. Pures Appl. (9) 86 (2006), no. 3, 271-291. MR2257732 (2007e:35279)

[19] E. Chasseigne, The Dirichlet problem for some nonlocal diffusion equations. Differential Integral Equations. 20 (2007), no. 12, 1389-1404. MR2377023(2009a:35106)

[20] R. Cont, P. Tankov, Financial modelling with jump processes. Chapman \& Hall/CRC Financial Mathematics Series (2004). MR2042661 (2004m:91004)

[21] J. Coville, L. Dupaigne, On a non-local equation arising in population dynamics. Proc. Roy. Soc. Edinburgh Sect. A 137 (2007), no. 4, 727-755. MR2345778(2008i:35139)

[22] H. Cramér, Sur un nouveau théorème limite de la théorie des probabilités. Actualités Scientifiques et industrielles $\mathbf{7 3 6}$ (1938), 5-23.

[23] M.G. Crandall, P.-L. Lions, Viscosity solutions of Hamilton-Jacobi equations. Trans. Amer. Math. Soc., 277 (1983), no. 1, 1-42. MR690039(85g:35029) 
[24] M.D. Donsker, S.R.S. Varadhan, Asymptotic evaluation of certain Markov process expectations for large time, Comm. Pure Appl. Math. 28 (1975), 1-47. MR0386024(52:6883)

[25] L.C. Evans, H. IshiI, A PDE approach to some asymptotic problems concerning random differential equations with small noise intensities, Ann. Inst. H. Poincaré 2 (1985) no. 1, 1-20. MR.781589 (86f:35183)

[26] L.C. Evans, P.E. Souganidis, A PDE approach to geometric optics for certain semilinear parabolic equations, Indiana Univ. Math. J. 38 (1989), no. 1, 141-172. MR982575 (90f:35093)

[27] W.H. Fleming, Exit probabilities and optimal stochastic control, Applied Math. and Opt. 4 (1978), 329-346. MR.512217 (80h:60100)

[28] J. Feng, T.G. KurTz, Large deviations for stochastic processes, Mathematical Surveys and Monographs, 131. American Mathematical Society, Providence, RI, 2006. MR2260560 (2009g:60034)

[29] M. Freiduin, Functional integration and partial differential equations. Annals of Mathematics Studies 109. Princeton University Press, Princeton, NJ, 1985. MR833742 (87g:60066)

[30] M. Freidlin, Limit theorems for large deviations and reaction-diffusion equations, Ann. Probab. 13 (1985), no. 3, 639-675. MR799415 (87a:35104)

[31] F. Den Hollander, Large deviations. Fields Institute Monographs, 14. American Mathematical Society, Providence, RI, 2000. MR.1739680(2001f:60028)

[32] A. Kolmogoroff, I. Petrovskit, N. Piskounov, Etude de l'équation de la quantité de la matière et son application à un problème biologique. Moscow Univ. Math. Bull. 1 (1937), $1-25$.

[33] P.-L. Lions, Generalized solutions of Hamilton-Jacobi equations. Research Notes in Mathematics, 69. Pitman (Advanced Publishing Program), Boston, Mass.-London, 1982. MR667669 (84a:49038)

[34] B. ØKsendal, A. Sulem, Applied stochastic control of jump diffusions. Universitext. Springer-Verlag, Berlin, 2005. MR2109687 (2005i:93003)

[35] B. Perthame, Transport equations in biology. Frontiers in Mathematics. Birkhaüser Verlag, Basel, 2007. MR2270822(2007j:35004)

[36] R.T. Rockafellar, Convex analysis. Princeton Mathematical Series, No. 28, Princeton University Press, Princeton, N.J., 1970. MR0274683 (43:445)

[37] S.R.S. Varadhan, Large deviations, Ann. Probab. 36 (2008), no. 2, 397-419. MR 2393987 (2009d:60070)

[38] A.D Wentzell, M.I Freidlin, Random Perturbations of Dynamical Systems. Springer, New-York, 1984. MR722136 (85a:60064)

Departamento de Matemáticas, Universidad Carlos iti Madrid, Avda. de la UniverSIDAD, 30, 28911 Leganés, SPAin

E-mail address: cbrandle@math.uc3m.es

Laboratoire de Mathématiques et Physique Théorique, UMr7350, Université F. Rabelais - Tours, Parc de Grandmont, 37200 Tours, France - and - Fédération de Recherche Denis Poisson - Fr2964 - Université d’Orléans \& Université F. Rabelais Tours

E-mail address: echasseigne@univ-tours.fr 